\title{
NEVADA TEST SITE \\ 2009 DATA REPORT: \\ GROUNDWATER MONITORING PROGRAM AREA 5 RADIOACTIVE WASTE MANAGEMENT SITE
}

January 2010

Prepared for:

U.S. Department of Energy

National Nuclear Security Administration

Nevada Site Office

Prepared by:

National Security Technologies, LLC Las Vegas, Nevada 


\section{DISCLAIMER}

Reference herein to any specific commercial product, process, or service by trade name, trademark, manufacturer, or otherwise, does not necessarily constitute or imply its endorsement, recommendation, or favoring by the U.S. Government or any agency thereof.

Available for sale to the public from:

U.S. Department of Commerce

National Technical Information Service

5301 Shawnee Road

Alexandria, VA 22312

Telephone: (800) 553-6847

Fax: (703) 605-6900

E-mail: orders@ntis.gov

Online ordering: http://www.ntis.gov/help/ordermethods.aspx

Available electronically at http://www.osti.gov/bridge

Available for a processing fee to the U.S. Department of Energy and its contractors, in paper, from:

U.S. Department of Energy

Office of Scientific and Technical Information

P.O. Box 62

Oak Ridge, TN 37831-0062

Telephone: (865) 576-8401

Fax: (865) 576-5728

E-mail: reports@adonis.osti.gov 


\section{NEVADA TEST SITE}

2009 DATA REPORT:

GROUNDWATER MONITORING PROGRAM AREA 5 RADIOACTIVE WASTE MANAGEMENT SITE

January 2010

Prepared for:

U.S. Department of Energy

National Nuclear Security Administration

Nevada Site Office

Prepared by:

National Security Technologies, LLC Las Vegas, Nevada 
THIS PAGE INTENTIONALLY LEFT BLANK 


\section{TABLE OF CONTENTS}

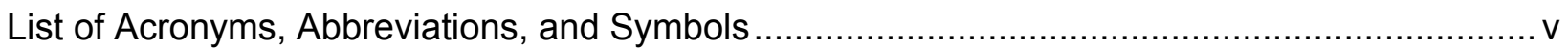

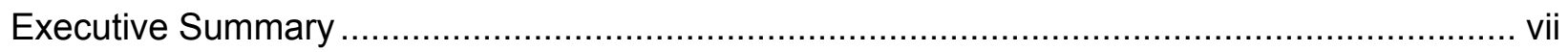

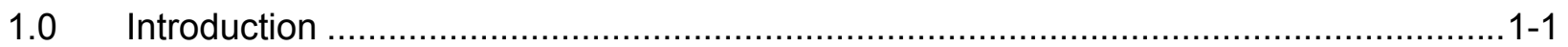

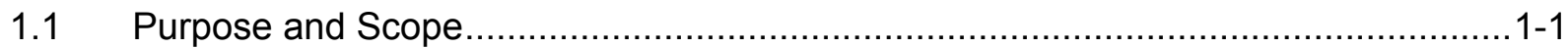

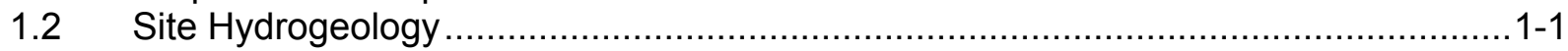

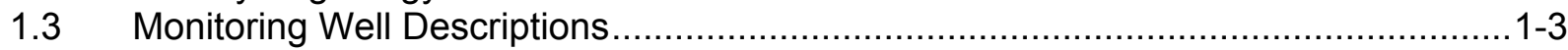

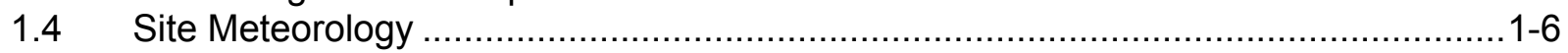

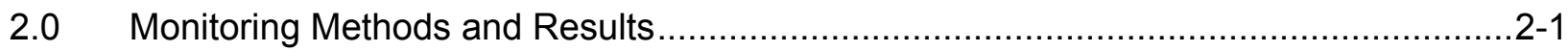

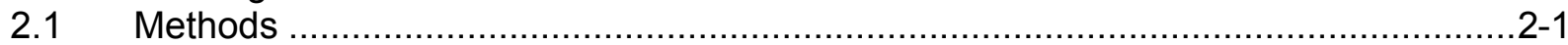

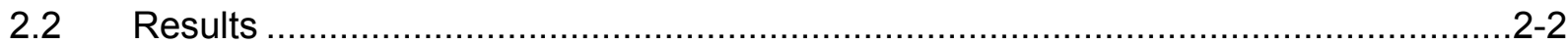

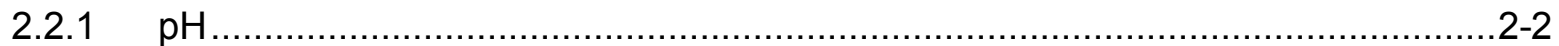

2.2.2 Specific Conductance.............................................................................

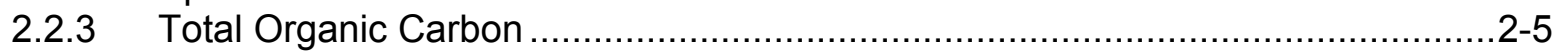

2.2.4 Total Organic Halides ........................................................................ $2-7$

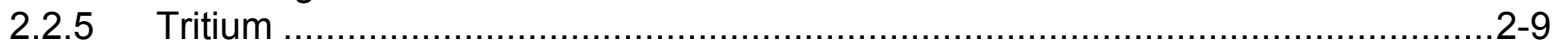

2.2.6 General Water Chemistry Parameters ................................................ 2-10

2.2.7 Groundwater Elevation .......................................................................

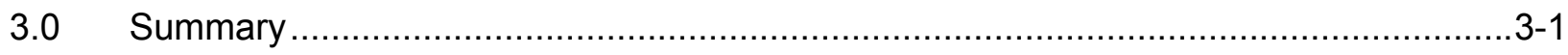

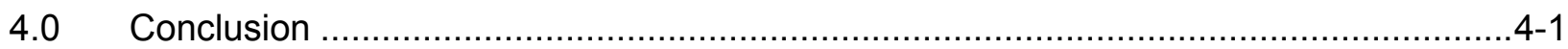

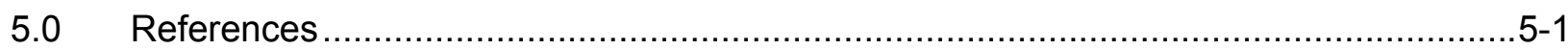

Appendix A - Cumulative Chronology for the Area 5 Radioactive Waste Management Site Groundwater Monitoring Program ............................................................................. A

Appendix B - Gradient/Velocity Calculations......................................................... B-1

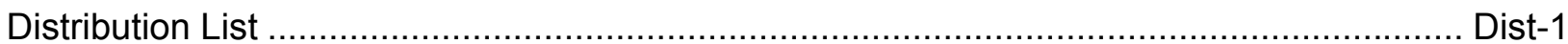




\section{LIST OF FIGURES}

Figure 1-1 Location of the Area 5 RWMS and Nevada Test Site within Nevada ................... 1-2

Figure 1-2 Location of Pilot Wells at the Area 5 RWMS .................................................1-2

Figure 1-3 Average Water Level Elevation at Groundwater Monitoring Wells in the Vicinity of the Area 5 RWMS (U.S. Geological Survey, 2009)................................................... 1-4

Figure 1-4 Groundwater Subbasins and Flow Directions in the Vicinity of the Area 5 RWMS .1-5

Figure 1-5 Monthly Precipitation at the Area 5 RWMS .................................................. 1-6

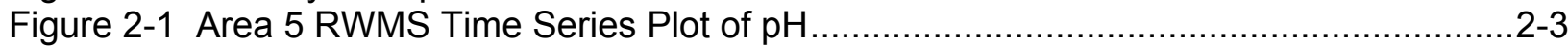

Figure 2-2 Area 5 RWMS Time Series Plot of SC .......................................................... $2-5$

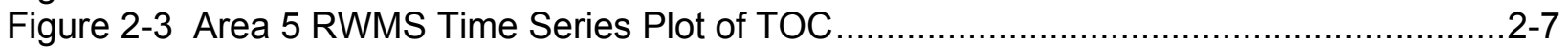

Figure 2-4 Area 5 RWMS Time Series Plot of TOX ..................................................... $2-8$

Figure 2-5 Area 5 RWMS Time Series Plot of Tritium .............................................. $2-10$

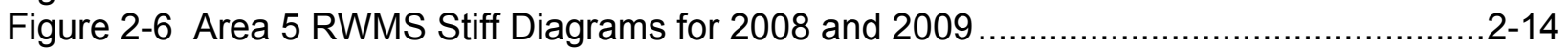

Figure 2-7 Area 5 RWMS Four-Year Piper Diagram .................................................. 2-15

Figure 2-8 Area 5 RWMS Time Series Plot of Groundwater Elevations ..............................2-19

\section{LIST OF TABLES}

Table 2-1 Investigation Levels of Indicator Parameters .................................................2

Table 2-2 Area 5 RWMS pH Values ............................................................................

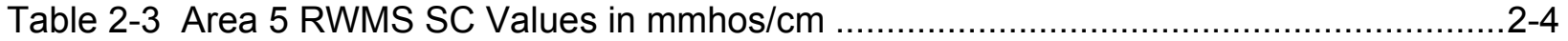

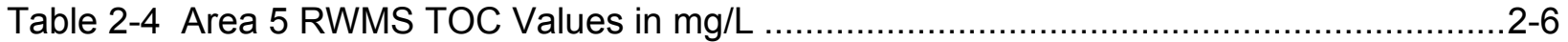

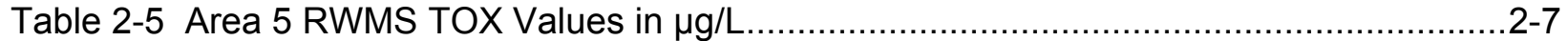

Table 2-6 Area 5 RWMS Tritium Values in pCi/L........................................................... $2-9$

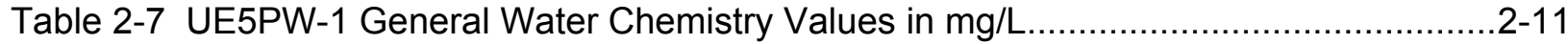

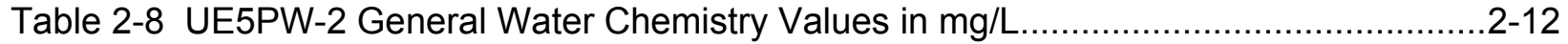

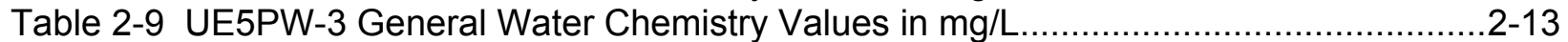

Table 2-10 Area 5 RWMS Groundwater Elevation Data................................................ 2-16

Table 2-11 2009 Area 5 RWMS Groundwater Flow Calculations .......................................2-18 


\section{LIST OF ACRONYMS, ABBREVIATIONS, AND SYMBOLS}

\begin{tabular}{|c|c|}
\hline AMSL & above mean sea level \\
\hline BN & Bechtel Nevada \\
\hline${ }^{\circ} \mathrm{C}$ & degrees Celsius \\
\hline $\mathrm{Ca}$ & calcium \\
\hline CFR & Code of Federal Regulations \\
\hline $\mathrm{Cl}$ & chloride \\
\hline $\mathrm{cm}$ & centimeter \\
\hline $\mathrm{cm} / \mathrm{yr}$ & centimeter per year \\
\hline DOE & U.S. Department of Energy \\
\hline $\mathrm{E}$ & easting \\
\hline${ }^{\circ} \mathrm{F}$ & degrees Fahrenheit \\
\hline $\mathrm{F}$ & fluoride \\
\hline $\mathrm{Fe}$ & iron \\
\hline $\mathrm{ft}$ & feet \\
\hline GW & groundwater \\
\hline $\mathrm{HCO}_{3}$ & bicarbonate \\
\hline IL & investigation level \\
\hline in. & inch \\
\hline in./year & inch per year \\
\hline $\mathrm{K}$ & potassium \\
\hline LCA & lower carbonate aquifer \\
\hline L & liter \\
\hline $\mathrm{m}$ & meter \\
\hline $\mathrm{m} / \mathrm{m}$ & meter change in water level elevation per meter change in gradient direction \\
\hline $\mathrm{m}^{3} / \mathrm{m}^{3}$ & void space volume (cubic meter) per total aquifer volume (cubic meter) \\
\hline MDC & minimum detectable concentration \\
\hline MDL & method detection limit \\
\hline $\mathrm{Mg}$ & magnesium \\
\hline $\mathrm{mg} / \mathrm{L}$ & milligram per liter \\
\hline $\mathrm{mmhos} / \mathrm{cm}$ & millimhos per centimeter \\
\hline Mn & manganese \\
\hline $\mathrm{N}$ & northing \\
\hline $\mathrm{Na}$ & sodium \\
\hline NDEP & Nevada Division of Environmental Protection \\
\hline NTS & Nevada Test Site \\
\hline $\mathrm{pCi} / \mathrm{L}$ & picocurie per liter \\
\hline RCRA & Resource Conservation and Recovery Act \\
\hline REECo & Reynolds Electrical and Engineering Company, Inc. \\
\hline RWMS & Radioactive Waste Management Site \\
\hline SC & specific conductance \\
\hline $\mathrm{SiO}_{2}$ & silicate \\
\hline $\mathrm{SO}_{4}$ & sulfate \\
\hline TOC & total organic carbon \\
\hline TOX & total organic halides \\
\hline$\mu \mathrm{g} / \mathrm{L}$ & microgram per liter \\
\hline
\end{tabular}


THIS PAGE INTENTIONALLY LEFT BLANK 


\section{EXECUTIVE SUMMARY}

This report is a compilation of the groundwater sampling results from the Area 5 Radioactive Waste Management Site (RWMS). The data have been collected since 1993 and include calendar year 2009 results. During 2009, groundwater at each of the three pilot wells was sampled on March 10, 2009, and August 18, 2009, and water levels at each of the three pilot wells were measured on February 17, May 6, August 17, and November 10, 2009. Groundwater samples were analyzed for the following indicators of contamination: $\mathrm{pH}$, specific conductance, total organic carbon, total organic halides, and tritium. Indicators of general water chemistry (cations and anions) were also measured. Results from all samples collected in 2009 were within the limits established by agreement with the Nevada Division of Environmental Protection for each analyte. These data indicate that there has been no measurable impact to the uppermost aquifer from the Area 5 RWMS.

There were no significant changes in measured groundwater parameters compared to previous years.

The report contains an updated cumulative chronology for the Area 5 RWMS Groundwater Monitoring Program and a brief description of the site hydrogeology. 
THIS PAGE INTENTIONALLY LEFT BLANK 


\subsection{INTRODUCTION}

\subsection{PURPOSE AND SCOPE}

This report is a compilation of the groundwater sampling results from three monitoring wells located near the Area 5 Radioactive Waste Management Site (RWMS) at the Nevada Test Site (NTS), Nye County, Nevada, and includes the results for calendar year 2009. The NTS is an approximately 3,561 square kilometer (1,375 square mile) restricted-access federal facility located approximately 105 kilometers (65 miles) northwest of Las Vegas, Nevada (Figure 1-1). Pilot Wells UE5PW-1, UE5PW-2, and UE5PW-3 are used to monitor the groundwater at the Area 5 RWMS (Figure 1-2). In addition to groundwater monitoring results, this report includes information regarding site hydrogeology, well construction, sample collection, and meteorological data measured at the Area 5 RWMS.

The disposal of low-level radioactive waste and mixed low-level radioactive waste at the Area 5 RWMS is regulated by U.S. Department of Energy (DOE) Order DOE O 435.1, "Radioactive Waste Management" (DOE, 2001). The disposal of mixed low-level radioactive waste is also regulated by the State of Nevada under the Resource Conservation and Recovery Act (RCRA) regulation Title 40 Code of Federal Regulations (CFR) Part 265, "Interim Status Standards for Owners and Operators of Hazardous Waste Treatment, Storage, and Disposal Facilities" (CFR, 1999). The format of this report was requested by the Nevada Division of Environmental Protection (NDEP) in a letter dated August 12, 1997. The appearance and arrangement of this document have been modified slightly since that date to provide additional information and to facilitate the readability of the document. The objective of this report is to satisfy any Area 5 RWMS reporting agreements between DOE and NDEP.

\subsection{SITE HYDROGEOLOGY}

The Area 5 RWMS is located in northern Frenchman Flat in the southeast portion of the NTS. Frenchman Flat is a topographically closed basin. Erosion of surrounding mountains has resulted in accumulation of thick, unsaturated, alluvial deposits above volcanic rocks within the basin (Bright et al., 2001). Alluvial and volcanic aquifers are present beneath the Area 5 RWMS and are believed to extend throughout much of the Frenchman Flat basin (Bechtel Nevada [BN], 2005). In this south-central portion of the NTS, a moderately thick volcanic confining unit, consisting of altered volcanic rocks, separates the shallow alluvial and volcanic aquifers from the underlying regional lower carbonate aquifer (LCA) (BN, 2005; Laczniak et al., 1996).

The groundwater type from the three monitoring wells (UE5PW-1, UE5PW-2, and UE5PW-3) is sodium-bicarbonate. This type of water is common in the upper aquifers in Frenchman Flat. UE5PW-1 and UE5PW-2 are completed in the alluvial aquifer, and UE5PW-3 is completed in the volcanic aquifer. Similar groundwater chemistry and water table elevations in UE5PW-1, UE5PW-2, and UE5PW-3 indicate that the alluvial and volcanic aquifers are locally connected near the Area 5 RWMS. 


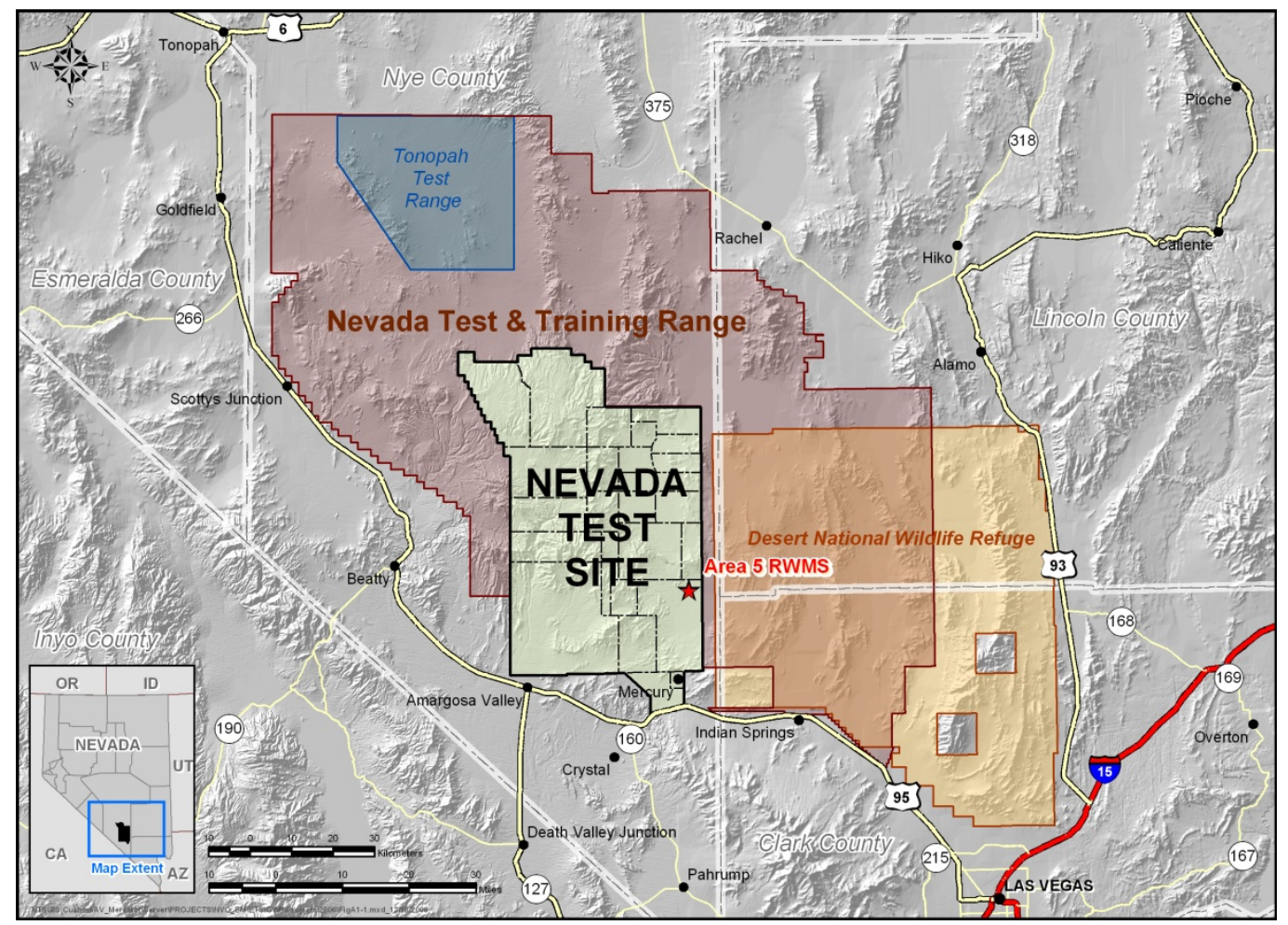

Figure 1-1 Location of the Area 5 RWMS and Nevada Test Site within Nevada

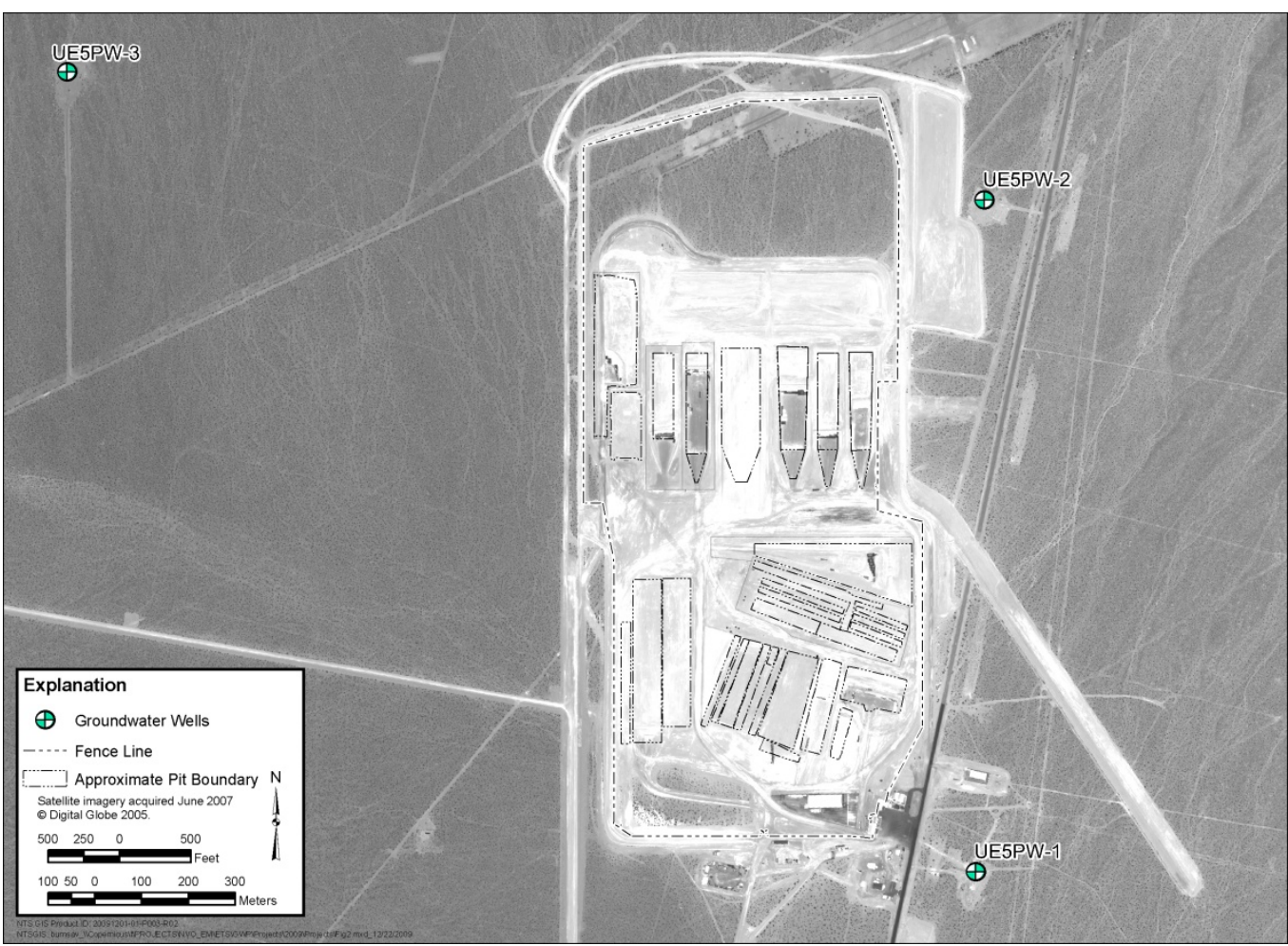

Figure 1-2 Location of Pilot Wells at the Area 5 RWMS 
Some vertical groundwater flow is thought to occur between the uppermost aquifers in Frenchman Flat and the underlying regional LCA, and is being studied by the Underground Test Area Sub-Project (Stoller-Navarro, 2006). Based on measured groundwater elevations above mean sea level (AMSL) (Figure 1-3), the lateral hydraulic gradient in the upper Frenchman Flat aquifer is very low. Lateral groundwater movement beneath Frenchman Flat primarily occurs within the deep carbonate aquifer and is generally from the northeast to southwest. It eventually discharges in Amargosa Valley and Ash Meadows in southwest Nevada and Death Valley in California (Figure 1-4) (Laczniak et al., 1996).

For more detailed descriptions of Area 5 RWMS site characteristics, refer to the report Revised Area 5 Radioactive Waste Management Site, Outline of a Comprehensive Groundwater Monitoring Program (BN, 1998).

\subsection{MONITORING WELL DESCRIPTIONS}

Pilot Wells UE5PW-1, UE5PW-2, and UE5PW-3 were drilled between March and November 1992, and the groundwater has been monitored since 1993. Each well is completed with a centralized 6.35-centimeter $(\mathrm{cm})(2.50$-inch [in.]) diameter stainless steel casing with an 18.3-meter $(\mathrm{m})$ (60-feet [ft]) dual-screen filter pack attached to the bottom of the casing. The borehole annulus below and around the screen is filled with 6/12 coarse mesh sand (Reynolds Electrical and Engineering Company, Inc. [REECo], 1994).

UE5PW-1 is $255.7 \mathrm{~m}$ (839 ft) deep from top of casing and is screened from $232.3 \mathrm{~m}$ (762 $\mathrm{ft}$ ) to $250.5 \mathrm{~m}$ (822 ft). UE5PW-1 is completed in alluvium. During 2009, the average water table depth below the top of the well casing was $235.79 \mathrm{~m}(773.59 \mathrm{ft})$, and the average water table elevation was $733.58 \mathrm{~m}(2,406.75 \mathrm{ft})$ AMSL.

UE5PW-2 is $280.3 \mathrm{~m}(919.5 \mathrm{ft})$ deep from top of casing and is screened from $253.0 \mathrm{~m} \mathrm{(830} \mathrm{ft)} \mathrm{to}$ $271.3 \mathrm{~m}$ (890 ft). UE5PW-2 is completed in alluvium. During 2009, the average water table depth below the top of the well casing was $256.42 \mathrm{~m}(841.27 \mathrm{ft})$, and the average water table elevation was $733.71 \mathrm{~m}(2,407.18 \mathrm{ft})$ AMSL.

UE5PW-3 is $291.1 \mathrm{~m}$ (955 ft) deep from top of casing and is screened from $267.6 \mathrm{~m}$ ( $878 \mathrm{ft}$ ) to $282.9 \mathrm{~m}$ (928 ft). UE5PW-3 is completed in volcanic rock. The alluvium volcanic rock contact is $188 \mathrm{~m}$ (617 ft) deep at UE5PW-3 (REECo, 1994). During 2009, the average water table depth below the top of the well casing was $271.53 \mathrm{~m}(890.84 \mathrm{ft})$, and the average water table elevation was $733.70 \mathrm{~m}(2,407.15 \mathrm{ft})$ AMSL. 


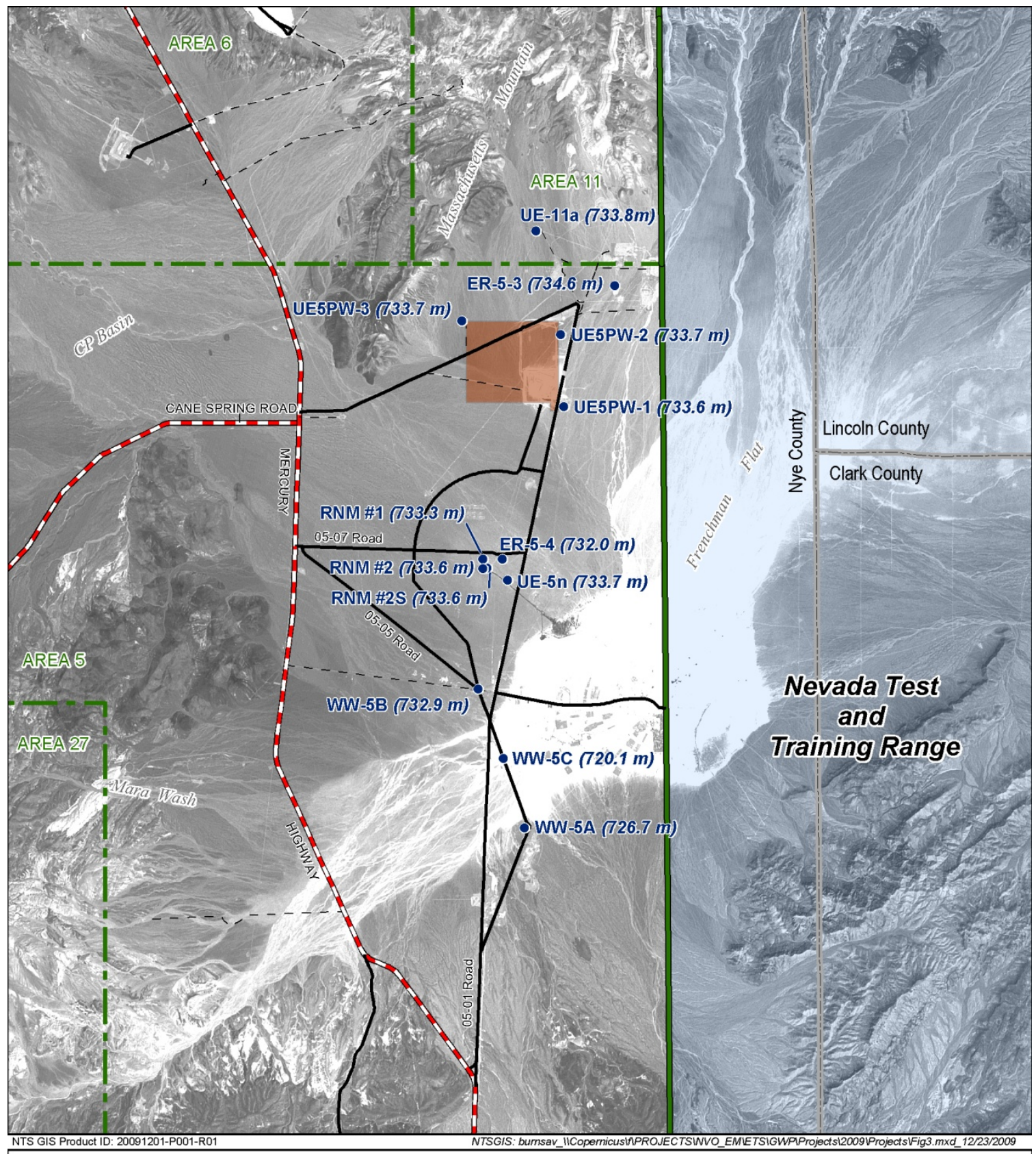

\section{Explanation}

- $\quad$ Monitoring Wells (Average Water Level - meters) Boundaries and Transportation

$\longrightarrow$ NTS Boundary

- - - NTS Operations Area Boundary

$=$ Secondary Highway

- Light-duty Road

- . - - Unimproved Road

Area 5 Radioactive Waste Management Complex

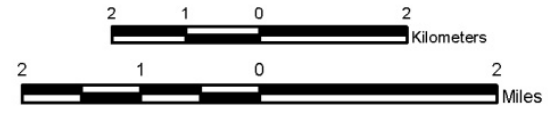

Nevada Central State Plane Projection (meters) North American Datum 1983

Background is 10-meter resolution SPOT imagery

Figure 1-3 Average Water Level Elevation at Groundwater Monitoring Wells in the Vicinity of the Area 5 RWMS (U.S. Geological Survey, 2009) 


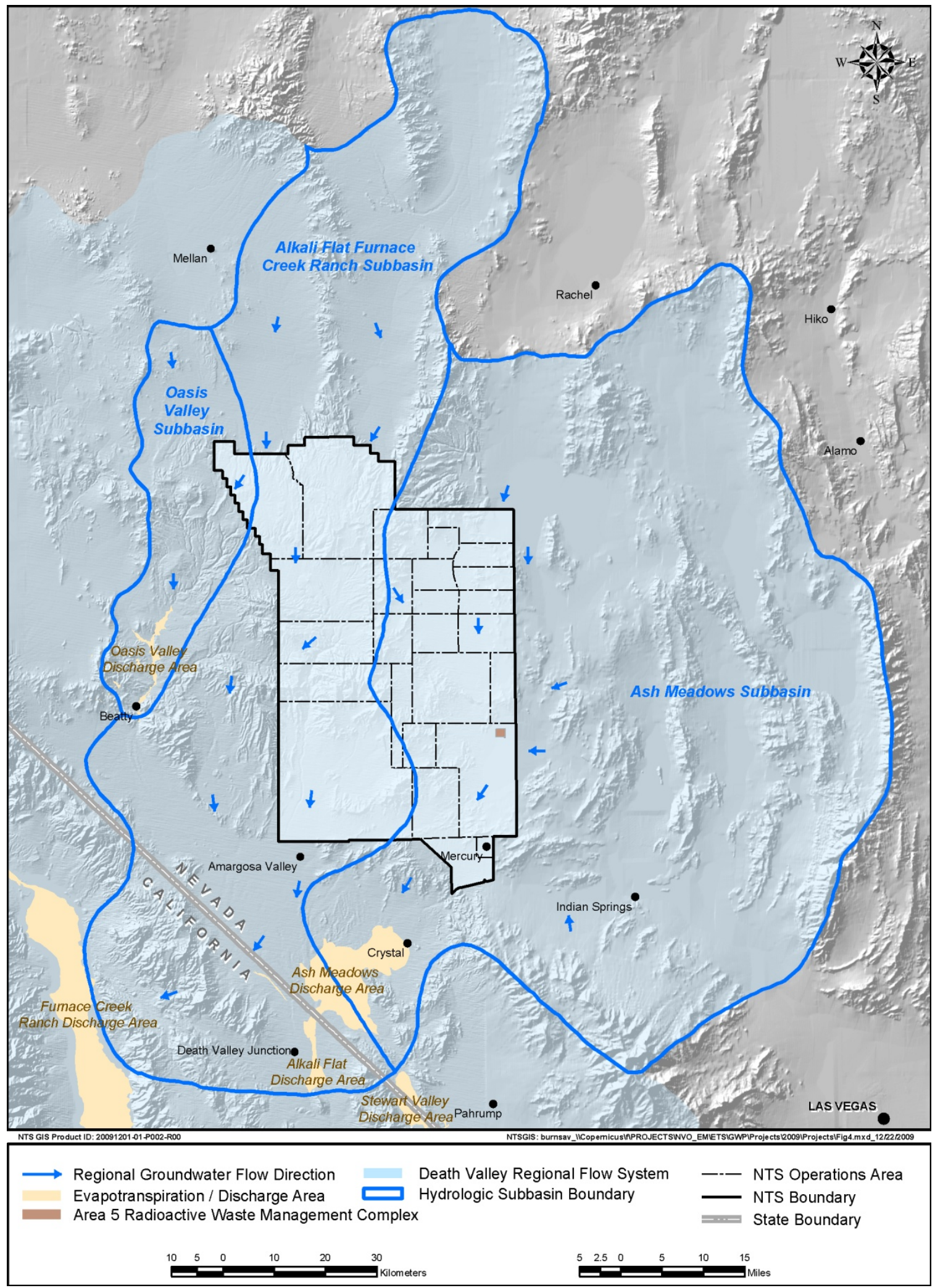

Figure 1-4 Groundwater Subbasins and Flow Directions in the Vicinity of the Area 5 RWMS 


\subsection{SITE METEOROLOGY}

Meteorological data are measured at the Area 5 RWMS. These data include temperature, relative humidity, barometric pressure, wind speed and direction, solar radiation, and precipitation. During 2009 the average daily temperature at $3 \mathrm{~m}$ height was 16.4 degrees Celsius $\left({ }^{\circ} \mathrm{C}\right)$ (61.5 degrees Fahrenheit $\left[{ }^{\circ} \mathrm{F}\right]$ ). The maximum observed temperature at $3 \mathrm{~m}$ height was $42.5^{\circ} \mathrm{C}\left(108.5^{\circ} \mathrm{F}\right)$ on July 18,2009 , and the minimum observed temperature at $3 \mathrm{~m}$ was $-12.9^{\circ} \mathrm{C}\left(8.8^{\circ} \mathrm{F}\right)$ on December 10,2009 . The maximum observed wind gust at $3 \mathrm{~m}$ was 20.9 meters/second (46.8 miles per hour) on October 28, 2009. The average annual precipitation measured at the Area 5 RWMS from 1994 through 2009 was $12.1 \mathrm{~cm}$ per year $(\mathrm{cm} / \mathrm{yr})$ (4.76 in./yr). There were $6.3 \mathrm{~cm}$ (2.48 in.) of precipitation at the Area 5 RWMS during 2009. During the 16-year measurement period, only the year 2002 had less precipitation than 2009. There were 33 days of measurable precipitation in 2009 at the Area 5 RWMS. The wettest month in 2009 was February, which had approximately 54 percent of the 2009 precipitation. Monthly precipitation at the Area 5 RWMS from January 1994 through December 2009 is provided in Figure 1-5.

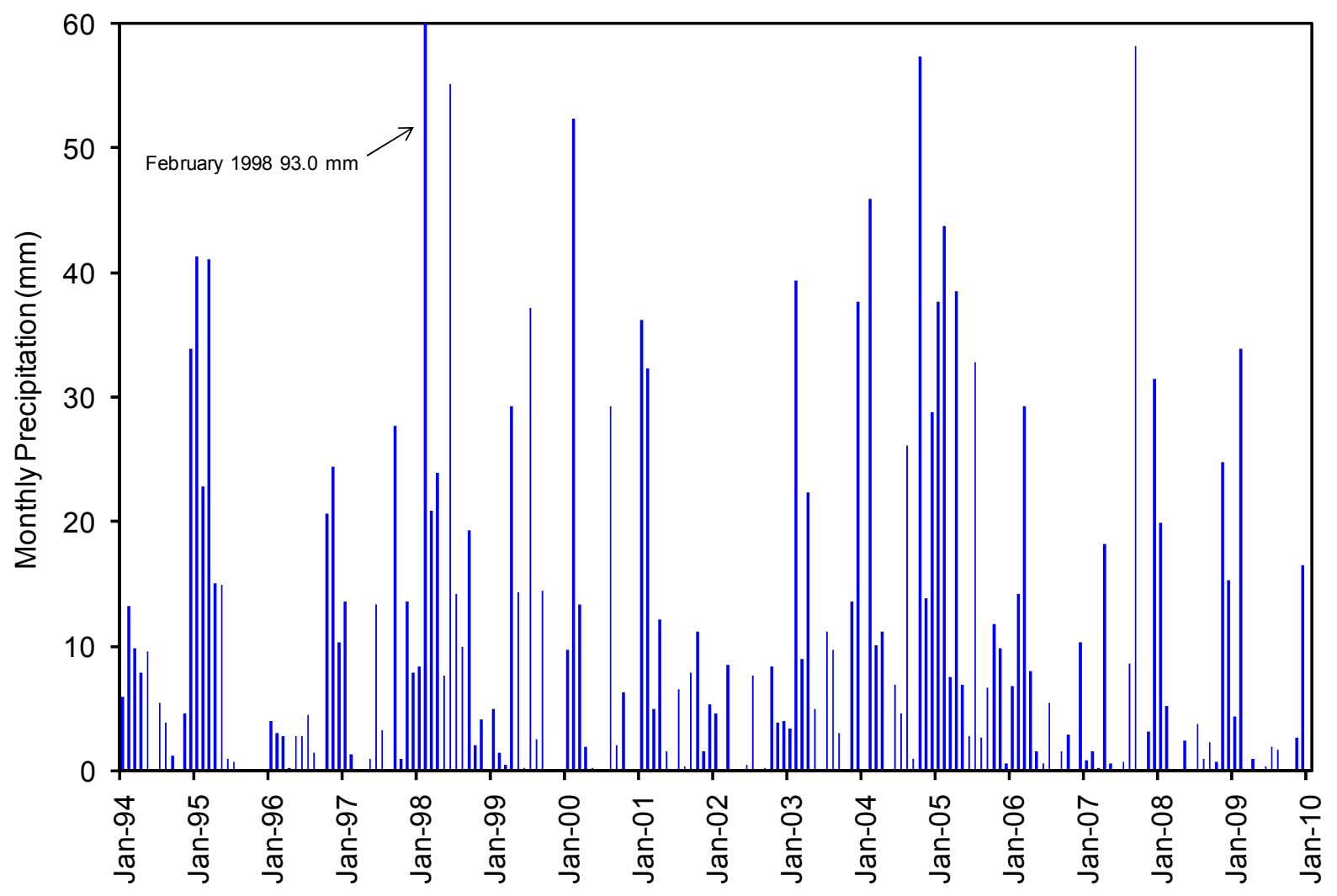

Figure 1-5 Monthly Precipitation at the Area 5 RWMS 


\subsection{MONITORING METHODS AND RESULTS}

The groundwater at the Area 5 RWMS pilot wells has been monitored since 1993 (see Appendix A). The Groundwater Monitoring Program has transitioned from monitoring all parameters required by 40 CFR 265 to a program that monitors parameters applicable to the Area 5 RWMS. The current monitoring program is modeled after the 40 CFR 265 Detection Monitoring Program.

\subsection{METHODS}

Samples are tested semiannually for the analytes listed below, which are divided into groups representing indicators of contamination and general water chemistry parameters:

Indicators of contamination:

- $\mathrm{pH}$

- Specific conductance (SC)

- Total organic carbon (TOC)

- Total organic halides (TOX)

- Tritium

General water chemistry parameters:

- Cations: calcium (Ca), iron (Fe), magnesium (Mg), manganese (Mn), potassium (K), sodium $(\mathrm{Na})$

- Anions: bicarbonate $\left(\mathrm{HCO}_{3}\right)$, sulfate $\left(\mathrm{SO}_{4}\right)$, chloride $(\mathrm{Cl})$, fluoride $(\mathrm{F})$

- Silicate $\left(\mathrm{SiO}_{2}\right)$

Investigation levels (ILs) for each analyte identified as an indicator of contamination were established by DOE and NDEP in 1998 (Table 2-1). Further groundwater analyses are required if an analyte's IL is exceeded. The intent of ILs is to replace the need for rigorous statistical analyses to identify contamination. Statistical analyses are not presented in this report, as agreed upon by NDEP in a letter dated April 17, 2000 (Liebendorfer, 2000). The ILs for pH and SC are based on the distributions of data collected from 1993 through 1996. Historic analyses for TOC, TOX, and tritium typically have concentration levels less than the method detection limit (MDL) or the minimum detectable concentration (MDC), so the ILs for TOC and TOX are set slightly above their MDLs or MDCs. The tritium IL is set at 2,000 picocuries per liter (pCi/L), which is 10 percent of the National Primary Drinking Water Standard of 20,000 pCi/L.

Wells UE5PW-1, UE5PW-2, and UE5PW-3 were sampled on March 10, 2009, and again on August 18, 2009. The current groundwater sampling procedure (National Security Technologies, LLC, 2006) was followed. Tritium samples were enriched prior to shipment to a contract laboratory for analysis. Analyses for tritium were conducted by General Engineering Laboratory for samples collected on March 10, 2009, and by Paragon Analytics Incorporated for samples collected on August 18, 2009. Analyses for TOX from samples collected on March 10, 2009, were conducted by American Radiation Services. All other analyses were conducted by Lionville Laboratory Incorporated. 
Table 2-1 Investigation Levels of Indicator Parameters

\begin{tabular}{|l|c|}
\hline Parameter & Investigation Level (IL) \\
\hline $\mathrm{pH}$ & $<7.6 \mathrm{or}>9.2$ \\
$\mathrm{SC}$ & $0.440 \mathrm{mmhos} / \mathrm{cm}^{\mathrm{a}}$ \\
TOC & $1 \mathrm{mg} / \mathrm{L}^{\mathrm{b}}$ \\
TOX & $50 \mu \mathrm{gg} / \mathrm{L}^{\mathrm{c}}$ \\
Tritium & $2,000 \mathrm{pCi} / \mathrm{L}$ \\
\hline
\end{tabular}

(a) $\mathrm{mmhos} / \mathrm{cm}=$ millimhos per centimeter

(b) $\mathrm{mg} / \mathrm{L}=$ milligrams per liter

(c) $\mu \mathrm{g} / \mathrm{L}=$ micrograms per liter

For TOC and TOX analysis, three replicate water samples were collected consecutively from each well for each analyte. This provides sufficient sample backups in case any sample result is above the analyte's IL. Well resampling is required if all three replicate water samples are above the analyte's IL. False detections of these analytes above their ILs and subsequent resampling of the wells have occurred in the past. No resampling was required in 2009.

\subsection{RESULTS}

This section lists the results for each of the five indicators of contamination, the general water chemistry parameters, and the groundwater elevation.

\subsection{1 pH}

The measured pH at each well remained within the ILs of 7.6 and 9.2 during 2009 (Table 2-2). The $2009 \mathrm{pH}$ values ranged from 8.00 to 8.45 and represent the stable $\mathrm{pH}$ reading obtained from each well just prior to sampling for other analytes. Measured $\mathrm{pH}$ has remained relatively stable throughout the entire monitoring period (Figure 2-1). No groundwater contamination is indicated by the $\mathrm{pH}$ monitoring results.

Table 2-2 Area 5 RWMS pH Values

\begin{tabular}{|cc|cc|cc|}
\hline \multicolumn{2}{|c|}{ UE5PW-1 } & \multicolumn{2}{c|}{ UE5PW-2 } & \multicolumn{2}{c|}{ UE5PW-3 } \\
\hline Date & pH & Date & pH & Date & pH \\
\hline $03 / 31 / 1993$ & 8.17 & $03 / 24 / 1993$ & 7.99 & $04 / 14 / 1993$ & 8.24 \\
$07 / 06 / 1993$ & 8.30 & $06 / 22 / 1993$ & 8.24 & $06 / 02 / 1993$ & 8.68 \\
$09 / 01 / 1993$ & 8.25 & $11 / 15 / 1993$ & 8.40 & $10 / 12 / 1993$ & 8.69 \\
$12 / 07 / 1993$ & 7.91 & $01 / 19 / 1994$ & 8.79 & $12 / 20 / 1993$ & 8.60 \\
$06 / 15 / 1994$ & 8.45 & \multicolumn{2}{c}{ No sample } & $05 / 24 / 1994$ & 8.87 \\
$08 / 01 / 1994$ & 8.28 & $06 / 07 / 1994$ & 8.81 & $08 / 08 / 1994$ & 8.77 \\
\multicolumn{2}{|c|}{ No sample } & $11 / 29 / 1994$ & 8.79 & $01 / 18 / 1995$ & 8.58 \\
$04 / 04 / 1995$ & 8.25 & $04 / 04 / 1995$ & 8.58 & $04 / 05 / 1995$ & 8.28 \\
$11 / 09 / 1995$ & 8.35 & $11 / 09 / 1995$ & 8.08 & $11 / 09 / 1995$ & 8.43 \\
$01 / 18 / 1996$ & 8.41 & $01 / 25 / 1996$ & 8.63 & $01 / 18 / 1996$ & 8.55 \\
$04 / 16 / 1996$ & 8.22 & $04 / 23 / 1996$ & 8.21 & $04 / 23 / 1996$ & 8.23 \\
No sample & & $04 / 30 / 1996$ & 8.15 & $04 / 30 / 1996$ & 8.15 \\
$10 / 02 / 1996$ & 8.18 & $10 / 02 / 1996$ & 8.28 & $10 / 02 / 1996$ & 8.18 \\
$11 / 20 / 1996$ & 8.25 & $11 / 20 / 1996$ & 8.16 & $11 / 20 / 1996$ & 8.13 \\
$04 / 16 / 1997$ & 8.33 & $04 / 16 / 1997$ & 8.40 & $04 / 16 / 1997$ & 8.25 \\
$11 / 05 / 1997$ & 8.30 & $11 / 05 / 1997$ & 8.17 & $11 / 05 / 1997$ & 8.22 \\
$05 / 13 / 1998$ & 8.31 & $05 / 13 / 1998$ & 8.37 & $05 / 13 / 1998$ & 8.34
\end{tabular}




\begin{tabular}{|cc|cc|cc|}
\hline \multicolumn{2}{|c|}{ UE5PW-1 } & \multicolumn{2}{c|}{ UE5PW-2 } & \multicolumn{2}{c|}{ UE5PW-3 } \\
\hline Date & pH & \multicolumn{2}{c|}{ Date } & pH & \multicolumn{2}{c|}{ Date } & pH \\
\hline $07 / 29 / 1998$ & 8.63 & \multicolumn{2}{c|}{ No sample sample } \\
$10 / 28 / 1998$ & 8.34 & $10 / 28 / 1998$ & 8.32 & $10 / 28 / 1998$ & 8.14 \\
$05 / 19 / 1999$ & 8.50 & $05 / 19 / 1999$ & 8.49 & $05 / 19 / 1999$ & 8.47 \\
$10 / 27 / 1999$ & 8.49 & $10 / 27 / 1999$ & 8.52 & $10 / 27 / 1999$ & 8.34 \\
$04 / 26 / 2000$ & 8.50 & $04 / 26 / 2000$ & 8.39 & $04 / 26 / 2000$ & 8.24 \\
$08 / 09 / 2000$ & 8.26 & $08 / 09 / 2000$ & 8.14 & $08 / 09 / 2000$ & 8.23 \\
$05 / 29 / 2001$ & 8.46 & $05 / 29 / 2001$ & 8.25 & $05 / 29 / 2001$ & 8.27 \\
$10 / 03 / 2001$ & 8.39 & $10 / 03 / 2001$ & 8.22 & $10 / 03 / 2001$ & 8.13 \\
$05 / 15 / 2002$ & 8.46 & $05 / 15 / 2002$ & 8.30 & $05 / 15 / 2002$ & 8.32 \\
$10 / 22 / 2002$ & 8.43 & $10 / 22 / 2002$ & 8.23 & $10 / 22 / 2002$ & 8.24 \\
$04 / 15 / 2003$ & 8.54 & $04 / 15 / 2003$ & 8.38 & $04 / 15 / 2003$ & 8.42 \\
$10 / 22 / 2003$ & 8.37 & $10 / 22 / 2003$ & 8.24 & $10 / 21 / 2003$ & 8.16 \\
$05 / 04 / 2004$ & 8.50 & $05 / 04 / 2004$ & 8.25 & $05 / 04 / 2004$ & 8.26 \\
$10 / 19 / 2004$ & 8.30 & $10 / 19 / 2004$ & 8.32 & $10 / 20 / 2004$ & 8.24 \\
$04 / 19 / 2005$ & 8.48 & $04 / 19 / 2005$ & 8.30 & $04 / 19 / 2005$ & 8.33 \\
$10 / 11 / 2005$ & 8.47 & $10 / 11 / 2005$ & 8.27 & $10 / 11 / 2005$ & 8.31 \\
$04 / 26 / 2006$ & 8.34 & $04 / 26 / 2006$ & 8.12 & $04 / 26 / 2006$ & 8.17 \\
$10 / 10 / 2006$ & 8.11 & $10 / 10 / 2006$ & 8.03 & $10 / 10 / 2006$ & 8.07 \\
$03 / 19 / 2007$ & 8.37 & $03 / 19 / 2007$ & 8.13 & $03 / 19 / 2007$ & 8.44 \\
$08 / 29 / 2007$ & 8.29 & $08 / 29 / 2007$ & 8.09 & $09 / 05 / 2007$ & 8.10 \\
$03 / 11 / 2008$ & 8.08 & $03 / 11 / 2008$ & 8.00 & $03 / 11 / 2008$ & 8.03 \\
$09 / 10 / 2008$ & 8.17 & $09 / 10 / 2008$ & 8.08 & $09 / 10 / 2008$ & 8.14 \\
$03 / 10 / 2009$ & 8.40 & $03 / 10 / 2009$ & 8.17 & $03 / 10 / 2009$ & 8.22 \\
$08 / 18 / 2009$ & 8.45 & $08 / 18 / 2009$ & 8.25 & $08 / 18 / 2009$ & 8.22 \\
\hline
\end{tabular}

Values before 05/19/1999 are means of multiple measurements and values from $05 / 19 / 1999$ to present are the stable $\mathrm{pH}$ value measured just prior to sampling.

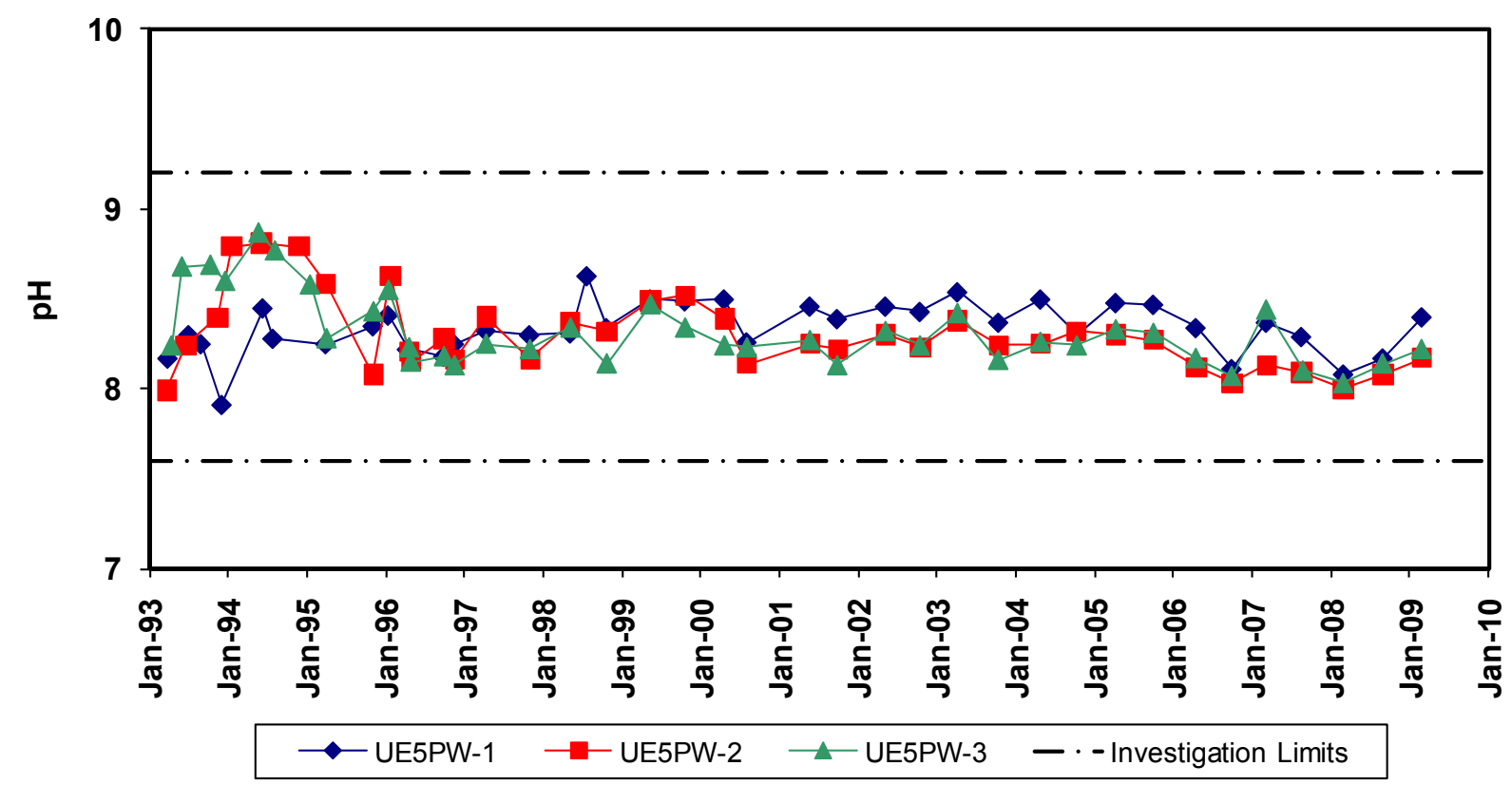

Figure 2-1 Area 5 RWMS Time Series Plot of pH 


\subsubsection{Specific Conductance}

The 2009 measured SC of water samples from each well remained below the IL of $0.440 \mathrm{mmhos} / \mathrm{cm}$ and ranged from 0.363 to $0.386 \mathrm{mmhos} / \mathrm{cm}$ (Table 2-3). SC values from each well have remained relatively stable throughout the entire monitoring period (Figure 2-2). No groundwater contamination is indicated by the SC monitoring results.

Table 2-3 Area 5 RWMS SC Values in $\mathrm{mmhos} / \mathrm{cm}$

\begin{tabular}{|c|c|c|c|c|c|}
\hline \multicolumn{2}{|c|}{ UE5PW-1 } & \multicolumn{2}{|c|}{ UE5PW-2 } & \multicolumn{2}{|c|}{ UE5PW-3 } \\
\hline Date & SC & Date & SC & Date & SC \\
\hline $03 / 31 / 1993$ & 0.401 & $03 / 24 / 1993$ & 0.371 & $04 / 14 / 1993$ & 0.383 \\
\hline 06/06/1993 & 0.391 & 06/22/1993 & 0.411 & 06/02/1993 & 0.382 \\
\hline 09/01/1993 & 0.391 & 11/15/1993 & 0.384 & 10/12/1993 & 0.376 \\
\hline 12/07/1993 & 0.383 & 01/19/1994 & 0.371 & $12 / 20 / 1993$ & 0.359 \\
\hline 06/15/1994 & 0.383 & 06/07/1994 & 0.363 & 05/24/1994 & 0.363 \\
\hline 08/01/1994 & 0.380 & \multicolumn{2}{|c|}{ No Sample } & 08/08/1994 & 0.367 \\
\hline \multicolumn{2}{|c|}{ No Sample } & $11 / 29 / 1994$ & 0.325 & 01/18/1995 & 0.338 \\
\hline 04/04/1995 & 0.320 & 04/04/1995 & 0.336 & 04/05/1995 & 0.347 \\
\hline $11 / 09 / 1995$ & 0.366 & 11/09/1995 & 0.348 & $11 / 09 / 1995$ & 0.352 \\
\hline 01/18/1996 & 0.360 & 01/25/1996 & 0.343 & 01/18/1996 & 0.355 \\
\hline 04/16/1996 & 0.363 & 04/23/1996 & 0.355 & 04/23/1996 & 0.363 \\
\hline \multicolumn{2}{|c|}{ No Sample } & 04/30/1996 & 0.356 & 04/30/1996 & 0.379 \\
\hline 10/02/1996 & 0.383 & 10/02/1996 & 0.363 & 10/02/1996 & 0.376 \\
\hline $11 / 20 / 1996$ & 0.374 & 11/20/1996 & 0.365 & 11/20/1996 & 0.378 \\
\hline 04/16/1997 & 0.385 & 04/16/1997 & 0.364 & 04/16/1997 & 0.376 \\
\hline $11 / 05 / 1997$ & 0.377 & $11 / 05 / 1997$ & 0.358 & $11 / 05 / 1997$ & 0.361 \\
\hline 05/13/1998 & 0.377 & 05/13/1998 & 0.356 & 05/13/1998 & 0.370 \\
\hline 07/29/1998 & 0.373 & \multicolumn{2}{|c|}{ No Sample } & \multicolumn{2}{|c|}{ No Sample } \\
\hline $10 / 28 / 1998$ & 0.380 & $10 / 28 / 1998$ & 0.358 & 10/28/1998 & 0.370 \\
\hline 05/19/1999 & 0.379 & 05/19/1999 & 0.351 & 05/19/1999 & 0.369 \\
\hline $10 / 27 / 1999$ & 0.370 & 10/27/1999 & 0.355 & 10/27/1999 & 0.370 \\
\hline $04 / 26 / 2000$ & 0.378 & $04 / 26 / 2000$ & 0.355 & 04/26/2000 & 0.369 \\
\hline 08/09/2000 & 0.378 & 08/09/2000 & 0.357 & 08/09/2000 & 0.370 \\
\hline 05/29/2001 & 0.377 & 05/29/2001 & 0.358 & 05/29/2001 & 0.371 \\
\hline $10 / 03 / 2001$ & 0.376 & $10 / 03 / 2001$ & 0.358 & $10 / 03 / 2001$ & 0.371 \\
\hline 05/15/2002 & 0.386 & $05 / 15 / 2002$ & 0.374 & 05/15/2002 & 0.384 \\
\hline $10 / 22 / 2002$ & 0.374 & $10 / 22 / 2002$ & 0.368 & $10 / 22 / 2002$ & 0.368 \\
\hline $04 / 15 / 2003$ & 0.372 & $04 / 15 / 2003$ & 0.355 & 04/15/2003 & 0.369 \\
\hline $10 / 22 / 2003$ & 0.376 & $10 / 22 / 2003$ & 0.357 & $10 / 21 / 2003$ & 0.373 \\
\hline 05/04/2004 & 0.378 & $05 / 04 / 2004$ & 0.361 & 05/04/2004 & 0.353 \\
\hline $10 / 19 / 2004$ & 0.372 & $10 / 19 / 2004$ & 0.352 & $10 / 20 / 2004$ & 0.365 \\
\hline 04/19/2005 & 0.377 & $04 / 19 / 2005$ & 0.359 & 04/19/2005 & 0.369 \\
\hline $10 / 11 / 2005$ & 0.368 & $10 / 11 / 2005$ & 0.352 & $10 / 11 / 2005$ & 0.364 \\
\hline $04 / 26 / 2006$ & 0.361 & $04 / 26 / 2006$ & 0.341 & 04/26/2006 & 0.357 \\
\hline $10 / 10 / 2006$ & 0.384 & $10 / 10 / 2006$ & 0.363 & $10 / 10 / 2006$ & 0.376 \\
\hline 03/19/2007 & 0.390 & 03/19/2007 & 0.330 & 03/19/2007 & 0.332 \\
\hline 08/29/2007 & 0.385 & $08 / 29 / 2007$ & 0.359 & 09/05/2007 & 0.378 \\
\hline $03 / 11 / 2008$ & 0.386 & $03 / 11 / 2008$ & 0.371 & $03 / 11 / 2008$ & 0.386 \\
\hline 09/10/2008 & 0.378 & 09/10/2008 & 0.360 & 09/10/2008 & 0.375 \\
\hline 03/10/2009 & 0.376 & 03/10/2009 & 0.363 & 03/10/2009 & 0.386 \\
\hline 08/18/2009 & 0.377 & 08/18/2009 & 0.363 & 08/18/2009 & 0.376 \\
\hline
\end{tabular}

Values before 05/19/1999 are means of multiple measurements and values from $05 / 19 / 1999$ to present are the stable SC value measured just prior to sampling. 


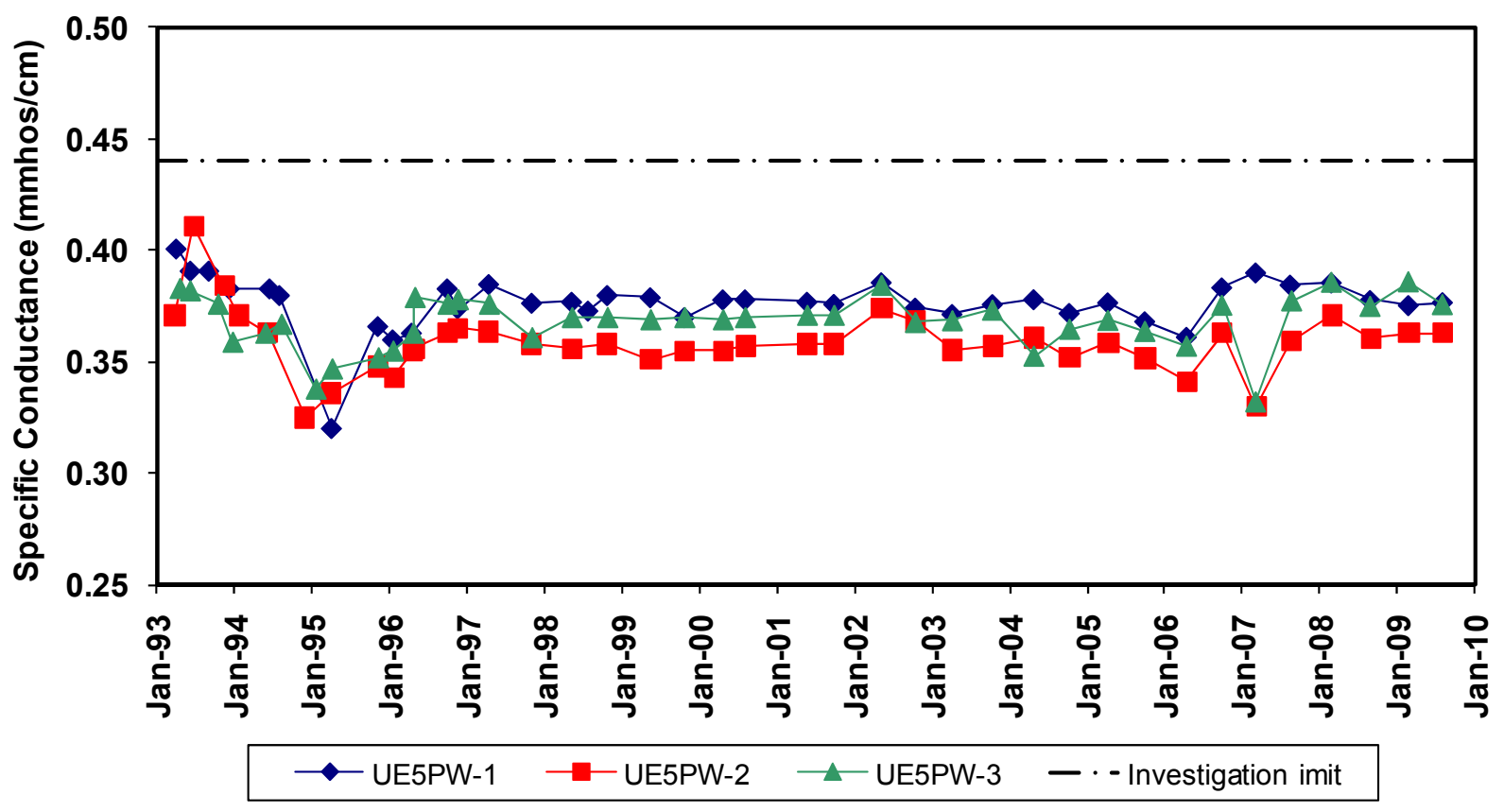

Figure 2-2 Area 5 RWMS Time Series Plot of SC

\subsubsection{Total Organic Carbon}

In 2009, three samples were collected consecutively from each well on both sample dates, and the averages of the three sample measurements are reported in Table 2-4. When sample TOC values fell below the sample's MDL of $0.5 \mathrm{mg} / \mathrm{L}$, then $0.5 \mathrm{mg} / \mathrm{L}$ was the value used to calculate the reported average. Values shown as $<0.5 \mathrm{mg} / \mathrm{L}$ indicate that all three sample results were less than the MDL. Samples collected from Well UE5PW-1 on March 10, 2009, had TOC results of $<0.5 \mathrm{mg} / \mathrm{L},<0.5 \mathrm{mg} / \mathrm{L}$, and $0.56 \mathrm{mg} / \mathrm{L}$; samples collected from Well UE5PW-1 on August 18, 2009 , had TOC results of $<0.5 \mathrm{mg} / \mathrm{L},<0.5 \mathrm{mg} / \mathrm{L}$, and $0.55 \mathrm{mg} / \mathrm{L}$; samples collected from Well UE5PW-2 on March 10, 2009, had TOC results of $<0.5 \mathrm{mg} / \mathrm{L}, 0.52 \mathrm{mg} / \mathrm{L}$, and $0.62 \mathrm{mg} / \mathrm{L}$; samples collected from Well UE5PW-2 on August 18, 2009, had TOC results of $<0.5 \mathrm{mg} / \mathrm{L}$, $<0.5 \mathrm{mg} / \mathrm{L}$, and $0.69 \mathrm{mg} / \mathrm{L}$; samples collected from Well UE5PW-3 on March 10, 2009, had all TOC results of <0.5 mg/L; and samples collected from Well UE5PW-3 on August 18, 2009, had TOC results of $<0.5 \mathrm{mg} / \mathrm{L},<0.5 \mathrm{mg} / \mathrm{L}$, and $0.57 \mathrm{mg} / \mathrm{L}$. All six TOC average values for 2008 are below the IL of $1 \mathrm{mg} / \mathrm{L}$.

TOC values have remained relatively low and stable throughout the monitoring period (Figure $2-3$ ). Most variation in TOC values is the result of variation in the MDL. No groundwater contamination is indicated by the TOC monitoring results. 
Table 2-4 Area 5 RWMS TOC Values in mg/L

\begin{tabular}{|c|c|c|c|c|c|}
\hline \multicolumn{2}{|c|}{ UE5PW-1 } & \multicolumn{2}{|c|}{ UE5PW-2 } & \multicolumn{2}{|c|}{ UE5PW-3 } \\
\hline Date & TOC & Date & TOC & Date & TOC \\
\hline 03/31/1993 & $<1.0$ & 03/24/1993 & $<1.0$ & $04 / 14 / 1993$ & $<1.0$ \\
\hline 07/06/1993 & $<1.0$ & $06 / 22 / 1993$ & $<1.0$ & 06/02/1993 & $<1.0$ \\
\hline 09/01/1993 & $<1.0$ & $11 / 15 / 1993$ & $<1.0$ & $10 / 12 / 1993$ & $<1.0$ \\
\hline 12/07/1993 & $<1.0$ & 01/19/1994 & $<1.0$ & $12 / 20 / 1993$ & $<1.0$ \\
\hline \multicolumn{2}{|c|}{ No Sample } & 06/07/1994 & $<1.0$ & \multicolumn{2}{|c|}{ No Sample } \\
\hline 08/01/1994 & $1.7^{\mathrm{a}}$ & $11 / 29 / 1994$ & $<1.0$ & 08/08/1994 & $<1.0$ \\
\hline 01/18/1995 & 0.20 & 01/18/1995 & 0.50 & 01/18/1995 & 0.23 \\
\hline 04/04/1995 & $<1.0$ & 04/04/1995 & $<1.0$ & 04/05/1995 & $<1.0$ \\
\hline 11/09/1995 & $<1.0$ & 11/20/1995 & $<1.0$ & 11/09/1995 & $<1.0$ \\
\hline 04/16/1996 & $<0.3$ & 04/30/1996 & $<0.3$ & 04/30/1996 & $<0.3$ \\
\hline 10/02/1996 & $<0.3$ & 10/02/1996 & $<0.3$ & $10 / 02 / 1996$ & $<0.3$ \\
\hline 11/20/1996 & $<0.3$ & $11 / 20 / 1996$ & $<0.3$ & $11 / 20 / 1996$ & $<0.3$ \\
\hline 04/16/1997 & $<0.3$ & 04/16/1997 & $<0.3$ & 04/16/1997 & $<0.3$ \\
\hline $11 / 05 / 1997$ & $<0.3$ & $11 / 05 / 1997$ & $<0.3$ & $11 / 05 / 1997$ & $<0.3$ \\
\hline 05/13/1998 & $<1.0$ & 05/13/1998 & $<1.0$ & 05/13/1998 & $<1.0$ \\
\hline 10/28/1998 & $<1.0$ & $10 / 28 / 1998$ & $<1.0$ & $10 / 28 / 1998$ & $<1.0$ \\
\hline 05/19/1999 & $<1.0$ & 05/19/1999 & $<1.0$ & 05/19/1999 & $<1.0$ \\
\hline 10/27/1999 & $<1.0$ & 10/27/1999 & 1.3 & 10/27/1999 & $<1.0$ \\
\hline \multicolumn{2}{|c|}{ No Sample } & $12 / 13 / 1999$ & $<0.5$ & \multicolumn{2}{|c|}{ No Sample } \\
\hline $04 / 26 / 2000$ & $0.98^{\mathrm{a}}$ & $04 / 26 / 2000$ & $0.60^{\mathrm{a}}$ & $04 / 26 / 2000$ & $1.3^{\mathrm{a}}$ \\
\hline $08 / 09 / 2000$ & $<0.5^{b}$ & 08/09/2000 & $<0.5^{\mathrm{b}}$ & $04 / 26 / 2000$ & $<0.5^{\mathrm{b}}$ \\
\hline $05 / 29 / 2001$ & $0.51^{\mathrm{b}}$ & $05 / 29 / 2001$ & $<0.5^{\mathrm{b}}$ & $05 / 29 / 2001$ & $0.53^{b}$ \\
\hline 10/03/2001 & $<0.5$ & $10 / 03 / 2001$ & $<0.5$ & $10 / 03 / 2001$ & $<0.5$ \\
\hline 05/15/2002 & $<0.5$ & 05/15/2002 & $<0.5$ & 05/15/2002 & $<0.5$ \\
\hline $10 / 22 / 2002$ & $<0.5$ & $10 / 22 / 2002$ & 0.55 & $10 / 22 / 2002$ & 0.58 \\
\hline $04 / 15 / 2003$ & 0.51 & $04 / 15 / 2003$ & 0.58 & $04 / 15 / 2003$ & 0.52 \\
\hline $10 / 22 / 2003$ & 0.64 & $10 / 22 / 2003$ & 0.68 & $10 / 21 / 2003$ & 0.62 \\
\hline 05/04/2004 & 0.55 & 05/04/2004 & $<0.5$ & 05/04/2004 & 0.58 \\
\hline $10 / 19 / 2004$ & 0.58 & $10 / 19 / 2004$ & 0.90 & $10 / 20 / 2004$ & 0.83 \\
\hline 04/19/2005 & 0.65 & $04 / 19 / 2005$ & 0.62 & $04 / 19 / 2005$ & 0.50 \\
\hline 10/11/2005 & 0.60 & $10 / 11 / 2005$ & 0.53 & $10 / 11 / 2005$ & $<0.5$ \\
\hline 04/26/2006 & $<0.5$ & 04/26/2006 & 0.97 & 04/26/2006 & 0.51 \\
\hline $10 / 10 / 2006$ & 0.80 & $10 / 10 / 2006$ & 1.12 & $10 / 10 / 2006$ & 0.52 \\
\hline 03/19/2007 & 0.62 & 03/19/2007 & 0.54 & 03/19/2007 & $<0.5$ \\
\hline 08/29/2007 & $<0.5$ & 08/29/2007 & $<0.5$ & 09/05/2007 & $<0.5$ \\
\hline 03/11/2008 & $<0.5$ & $03 / 11 / 2008$ & $<0.5$ & $03 / 11 / 2008$ & $<0.5$ \\
\hline 09/10/2008 & 0.537 & 09/10/2008 & 0.56 & 09/10/2008 & $<0.5$ \\
\hline 03/10/2009 & 0.520 & 03/10/2009 & 0.55 & 03/10/2009 & $<0.5$ \\
\hline $08 / 18 / 2009$ & 0.547 & $08 / 18 / 2009$ & 0.56 & $08 / 18 / 2009$ & 0.52 \\
\hline
\end{tabular}

(a) Determined to be a false positive through resampling

(b) Multiple laboratories used; this value is average of Lionville Laboratory only 


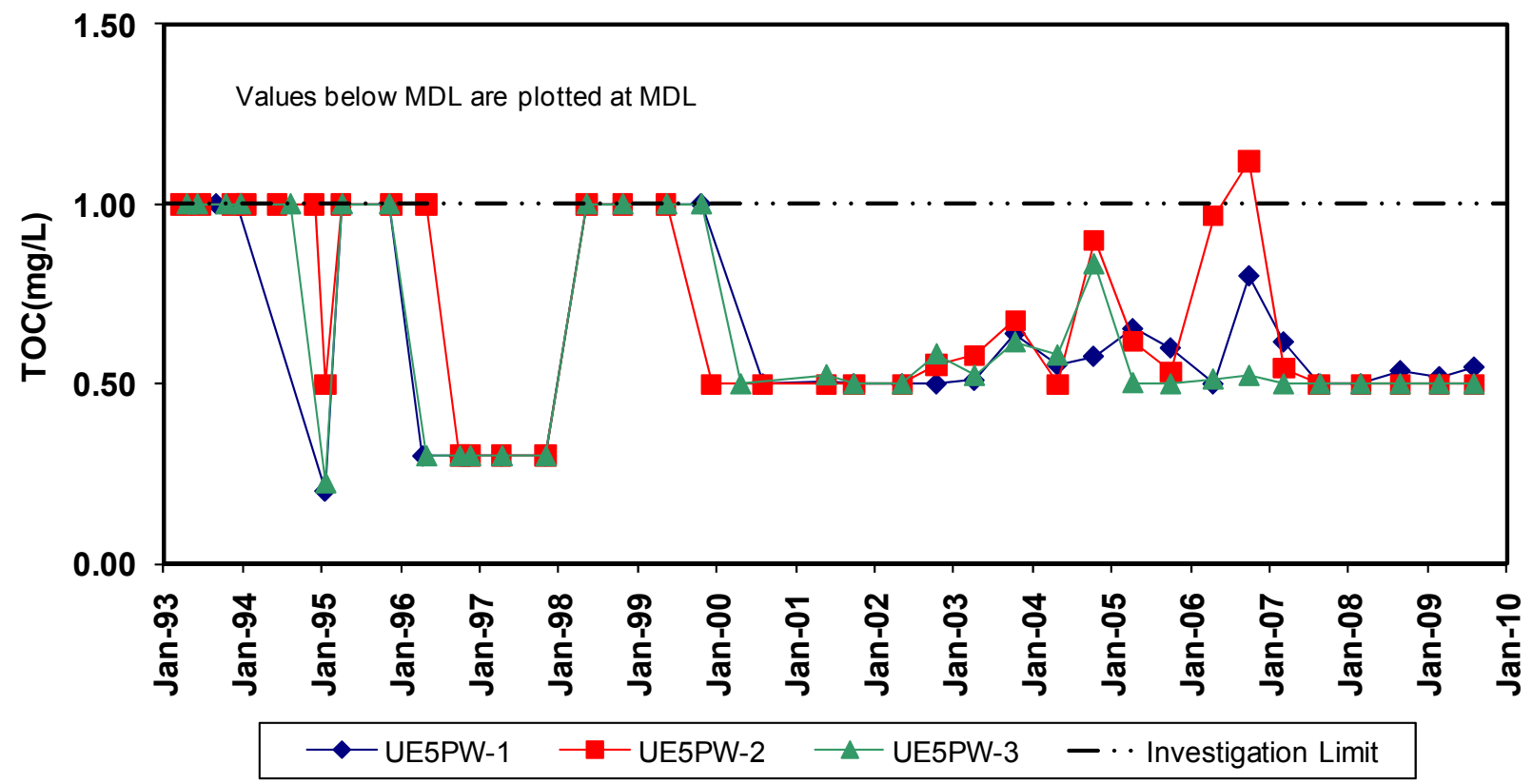

Figure 2-3 Area 5 RWMS Time Series Plot of TOC

\subsubsection{Total Organic Halides}

All 2009 TOX results are below the detection limit and below the IL of $50 \mu \mathrm{g} / \mathrm{L}$ (Table 2-5). In 2009 , three samples were collected from each well on each sample date, and the averages of the three sample results are reported in Table 2-5. When sample TOX values fell below the sample's MDL, the MDL was used to calculate the reported average. Values in Table 2-5 preceded by a less than symbol $(<)$ indicate that all three samples were less than the MDL. The MDL for samples collected on March 10, 2009, was $5 \mu \mathrm{g} / \mathrm{L}$ and the MDL for samples collected on August 18,2009 , was $7.7 \mu \mathrm{g} / \mathrm{L}$. Variation in MDL values are due to the analysis being done by different contract laboratories.

TOX values have remained relatively stable and below the IL throughout the monitoring period (Figure 2-4). No groundwater contamination is indicated by the TOX results.

Table 2-5 Area 5 RWMS TOX Values in $\mu \mathrm{g} / \mathrm{L}$

\begin{tabular}{|c|c|c|c|c|c|}
\hline \multicolumn{2}{|c|}{ UE5PW-1 } & \multicolumn{2}{|c|}{ UE5PW-2 } & \multicolumn{2}{|c|}{ UE5PW-3 } \\
\hline Date & TOX & Date & TOX & Date & TOX \\
\hline 03/31/1993 & 17 & 03/24/1993 & 23 & $04 / 14 / 1993$ & $<10$ \\
\hline 07/06/1993 & $<10$ & 06/22/1993 & $<10$ & 06/02/1993 & 13 \\
\hline 09/01/1993 & 13 & 11/15/1993 & $<10$ & 10/12/1993 & $<10$ \\
\hline 12/07/1993 & $<10$ & 01/19/1994 & $<10$ & $12 / 20 / 1993$ & $<10$ \\
\hline 06/15/1994 & $<10$ & 06/07/1994 & $<10$ & \multicolumn{2}{|c|}{ No Sample } \\
\hline 08/01/1994 & 11 & $11 / 29 / 1994$ & 13 & 08/08/1994 & $<10$ \\
\hline 01/18/1995 & $<10$ & 01/18/1995 & $<10$ & 01/18/1995 & $<10$ \\
\hline 04/04/1995 & $<10$ & 04/04/1995 & $<10$ & 04/05/1995 & $<10$ \\
\hline 11/09/1995 & $<40$ & 11/09/1995 & $<40$ & 11/09/1995 & $<40$ \\
\hline 04/16/1996 & $<40$ & 04/30/1996 & $<40$ & 04/30/1996 & $<40$ \\
\hline \multicolumn{2}{|c|}{ No Sample } & 10/02/1996 & $<20$ & $10 / 02 / 1996$ & $<20$ \\
\hline $11 / 20 / 1996$ & $<20$ & $11 / 20 / 1996$ & $<20$ & $11 / 20 / 1996$ & $<20$ \\
\hline
\end{tabular}




\begin{tabular}{|c|c|c|c|c|c|}
\hline 04/16/1997 & $<20$ & 04/16/1997 & $<20$ & 04/16/1997 & $<20$ \\
\hline $11 / 05 / 1997$ & $<20$ & $11 / 05 / 1997$ & $<20$ & $11 / 05 / 1997$ & $<20$ \\
\hline 05/13/1998 & $391^{a}$ & 05/13/1998 & $843^{a}$ & 05/13/1998 & $1000^{\mathrm{a}}$ \\
\hline 07/29/1998 & $<5$ & \multicolumn{2}{|c|}{ No Sample } & \multicolumn{2}{|c|}{ No Sample } \\
\hline 10/28/1998 & $<5$ & 10/29/1998 & $<5$ & 10/29/1998 & $<5$ \\
\hline 05/19/1999 & $<5$ & 05/19/1999 & $<5$ & 05/19/1999 & $<5$ \\
\hline 10/27/1999 & $<5$ & 10/27/1999 & $<5$ & 10/27/1999 & 7 \\
\hline $04 / 26 / 2000$ & $72^{a}$ & $04 / 26 / 2000$ & $59^{a}$ & $04 / 26 / 2000$ & $57^{a}$ \\
\hline 08/09/2000 & $92^{\mathrm{a}, \mathrm{b}}$ & 08/09/2000 & $73^{a, b}$ & 08/09/2000 & $83^{a, b}$ \\
\hline 05/29/2001 & $<12.7^{\mathrm{b}}$ & 05/29/2001 & $<12^{b}$ & 05/29/2001 & $<12^{\mathrm{b}}$ \\
\hline $10 / 03 / 2001$ & $<6.1$ & $10 / 03 / 2001$ & $<5.8$ & $10 / 03 / 2001$ & $<5.2$ \\
\hline $05 / 15 / 2002$ & $<5.2$ & $05 / 15 / 2002$ & 5.4 & 05/15/2002 & $<5.2$ \\
\hline $10 / 22 / 2002$ & $<5.2$ & $10 / 22 / 2002$ & $<5.2$ & $10 / 22 / 2002$ & $<5.2$ \\
\hline $04 / 15 / 2003$ & $<5.2$ & $04 / 15 / 2003$ & $<5.2$ & $04 / 15 / 2003$ & $<5.2$ \\
\hline $10 / 22 / 2003$ & $<5.2$ & $10 / 22 / 2003$ & 5.5 & $10 / 21 / 2003$ & $<5.2$ \\
\hline 05/04/2004 & $<5.2$ & $05 / 04 / 2004$ & $<5.2$ & $05 / 04 / 2004$ & $<5.2$ \\
\hline 10/19/2004 & $<5.2$ & $10 / 19 / 2004$ & $<5.2$ & $10 / 20 / 2004$ & $<5.2$ \\
\hline 04/19/2005 & $<5$ & 04/19/2005 & $<5$ & 04/19/2005 & $<5$ \\
\hline $10 / 11 / 2005$ & 5.2 & $10 / 11 / 2005$ & 6.5 & $10 / 11 / 2005$ & $<5$ \\
\hline 04/26/2006 & 7.3 & 04/26/2006 & 5.8 & 04/26/2006 & 7.4 \\
\hline 10/10/2006 & $<5.1$ & $10 / 10 / 2006$ & $<5$ & $10 / 10 / 2006$ & $<5$ \\
\hline 03/19/2007 & $<5.2$ & 03/19/2007 & $<5.2$ & 03/19/2007 & $<5.2$ \\
\hline 08/29/2007 & $<5.2$ & 08/29/2007 & $<5.2$ & 09/05/2007 & $<5.2$ \\
\hline 03/11/2008 & $<5.2$ & 03/11/2008 & $<5.2$ & 03/11/2008 & $<5.2$ \\
\hline 09/10/2008 & $<5.2$ & 09/10/2008 & 5.9 & 09/10/2008 & 8.9 \\
\hline 03/10/2009 & $<5$ & 03/10/2009 & $<5$ & 03/10/2009 & $<5$ \\
\hline 08/18/2009 & $<7.7$ & 08/18/2009 & $<7.7$ & 08/18/2009 & $<7.7$ \\
\hline
\end{tabular}

(a) Determined to be a false positive through resampling

(b) Multiple laboratories used; this value is average of Lionville Laboratory only

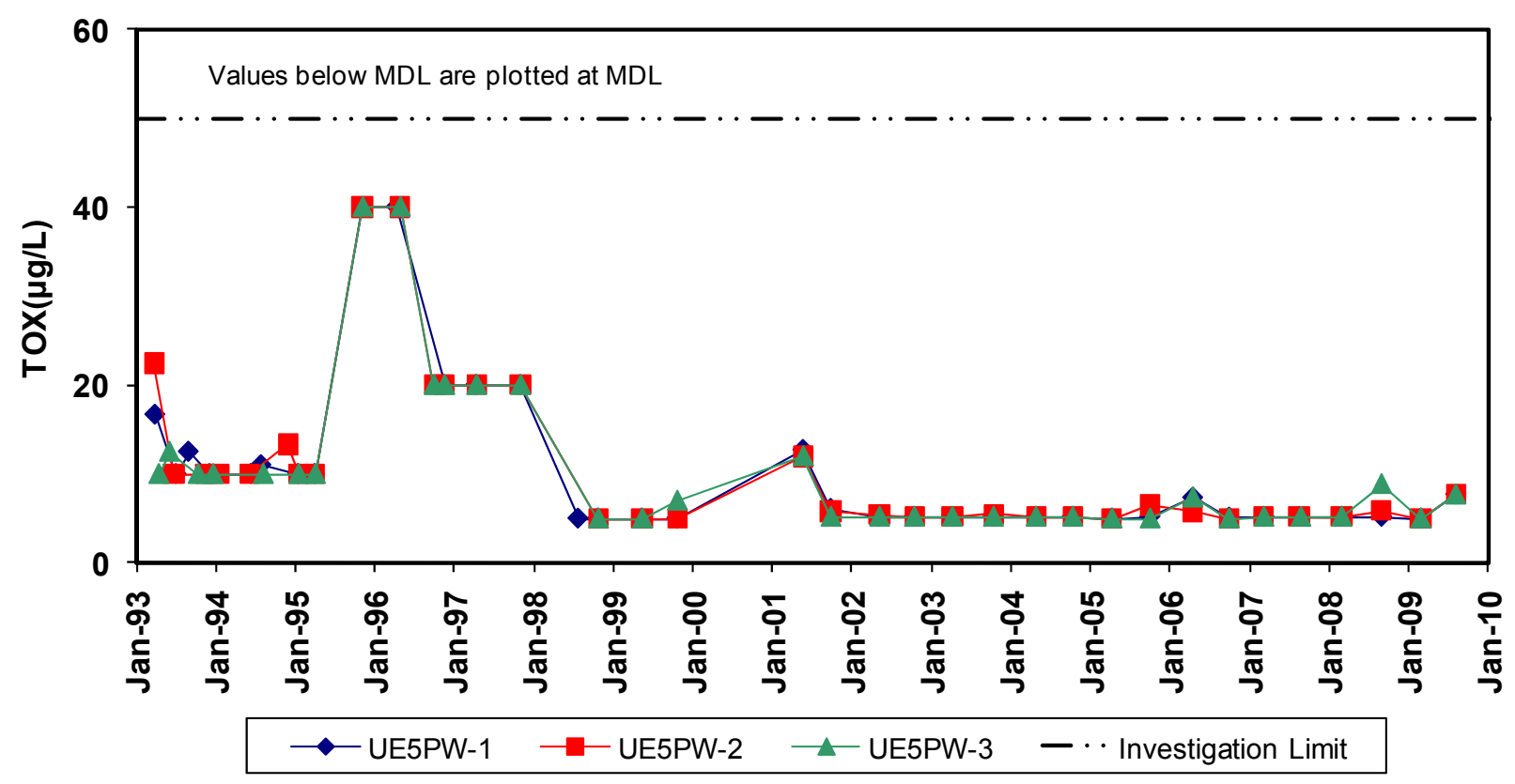

Figure 2-4 Area 5 RWMS Time Series Plot of TOX 


\subsubsection{Tritium}

All tritium results from 2009 water samples were below the IL of 2,000 pCi/L and below the laboratory MDC of approximately $20 \mathrm{pCi} / \mathrm{L}$ (Table 2-6). In 2009, duplicate samples were collected from each well on each sample date. Table 2-6 reports the average of these two results. Tritium values have remained relatively stable and below the IL and MDC throughout the monitoring period (Figure 2-5). No groundwater contamination is indicated by the tritium results.

Table 2-6 Area 5 RWMS Tritium Values in pCi/L

\begin{tabular}{|c|c|c|c|c|c|}
\hline \multicolumn{2}{|c|}{ UE5PW-1 } & \multicolumn{2}{|c|}{ UE5PW-2 } & \multicolumn{2}{|c|}{ UE5PW-3 } \\
\hline Date & Tritium & Date & Tritium & Date & Tritium \\
\hline 03/31/1993 & 0.442 & 03/24/1993 & -4.28 & 04/14/1993 & 1.96 \\
\hline 12/07/1993 & -1.58 & $11 / 15 / 1993$ & 32.2 & 06/02/1993 & -2.74 \\
\hline No $S$ & & 01/19/1994 & 3.69 & $12 / 20 / 1993$ & -0.459 \\
\hline 06/15/1994 & -2.04 & 06/07/1994 & 1.29 & 05/24/1994 & 1.13 \\
\hline 08/01/1994 & 1.86 & $11 / 29 / 1994$ & 0.015 & 08/08/1994 & 1.04 \\
\hline 04/04/1995 & 2.80 & 04/04/1995 & -0.920 & 04/05/1995 & 1.50 \\
\hline 04/16/1996 & -1.72 & 04/30/1996 & -1.91 & 04/30/1996 & -2.29 \\
\hline 04/16/1997 & 3.15 & 04/16/1997 & 0.189 & 04/16/1997 & 3.69 \\
\hline 05/13/1998 & -2.35 & 05/13/1998 & -1.95 & 05/13/1998 & -4.71 \\
\hline 10/28/1998 & -1.09 & 10/28/1998 & -1.85 & $10 / 28 / 1998$ & -8.25 \\
\hline 05/19/1999 & 5.17 & 05/19/1999 & 4.24 & 05/19/1999 & 4.60 \\
\hline 10/27/1999 & -1.36 & 10/27/1999 & -3.37 & 10/27/1999 & 1.08 \\
\hline 04/26/2000 & -2.56 & $04 / 26 / 2000$ & 1.17 & $04 / 26 / 2000$ & -0.080 \\
\hline 08/09/2000 & -1.48 & 08/09/2000 & 6.97 & 08/09/2000 & 4.35 \\
\hline 05/29/2001 & -1.90 & 05/29/2001 & -11.5 & 05/29/2001 & -12.4 \\
\hline 10/03/2001 & -2.93 & $10 / 03 / 2001$ & -2.82 & $10 / 03 / 2001$ & 2.46 \\
\hline 05/15/2002 & -2.82 & $05 / 15 / 2002$ & 0.150 & 05/15/2002 & -3.26 \\
\hline $10 / 22 / 2002$ & -4.15 & $10 / 22 / 2002$ & 0.113 & $10 / 22 / 2002$ & -1.17 \\
\hline 04/15/2003 & -1.13 & $04 / 15 / 2003$ & -5.22 & $04 / 15 / 2003$ & 1.62 \\
\hline $10 / 22 / 2003$ & 0.952 & $10 / 22 / 2003$ & 11.4 & $10 / 21 / 2003$ & 0.405 \\
\hline 05/04/2004 & -2.69 & 05/04/2004 & -6.17 & $05 / 04 / 2004$ & -6.04 \\
\hline 10/19/2004 & -1.50 & $10 / 19 / 2004$ & -10.0 & $10 / 20 / 2004$ & -6.39 \\
\hline 04/19/2005 & 3.67 & 04/19/2005 & 3.76 & 04/19/2005 & 3.56 \\
\hline 10/11/2005 & 8.83 & $10 / 11 / 2005$ & 5.24 & $10 / 11 / 2005$ & -4.78 \\
\hline $04 / 26 / 2006$ & 0.480 & $04 / 26 / 2006$ & -2.70 & $04 / 26 / 2006$ & -6.71 \\
\hline $10 / 10 / 2006$ & 7.42 & $10 / 10 / 2006$ & 9.35 & $10 / 10 / 2006$ & 13.75 \\
\hline 03/19/2007 & -10.33 & 03/19/2007 & -7.96 & 03/19/2007 & -4.15 \\
\hline 08/29/2007 & -7.25 & 08/29/2007 & -5.61 & 09/05/2007 & -5.60 \\
\hline 03/11/2008 & 5.33 & $03 / 11 / 2008$ & 7.63 & 03/11/2008 & -1.41 \\
\hline 09/10/2008 & 4.53 & 09/10/2008 & -2.03 & 09/10/2008 & -4.98 \\
\hline 03/10/2009 & 5.36 & 03/10/2009 & 11.80 & 03/10/2009 & -3.77 \\
\hline 08/18/2009 & 3.38 & 08/18/2009 & 1.62 & 08/18/2009 & 11.90 \\
\hline
\end{tabular}




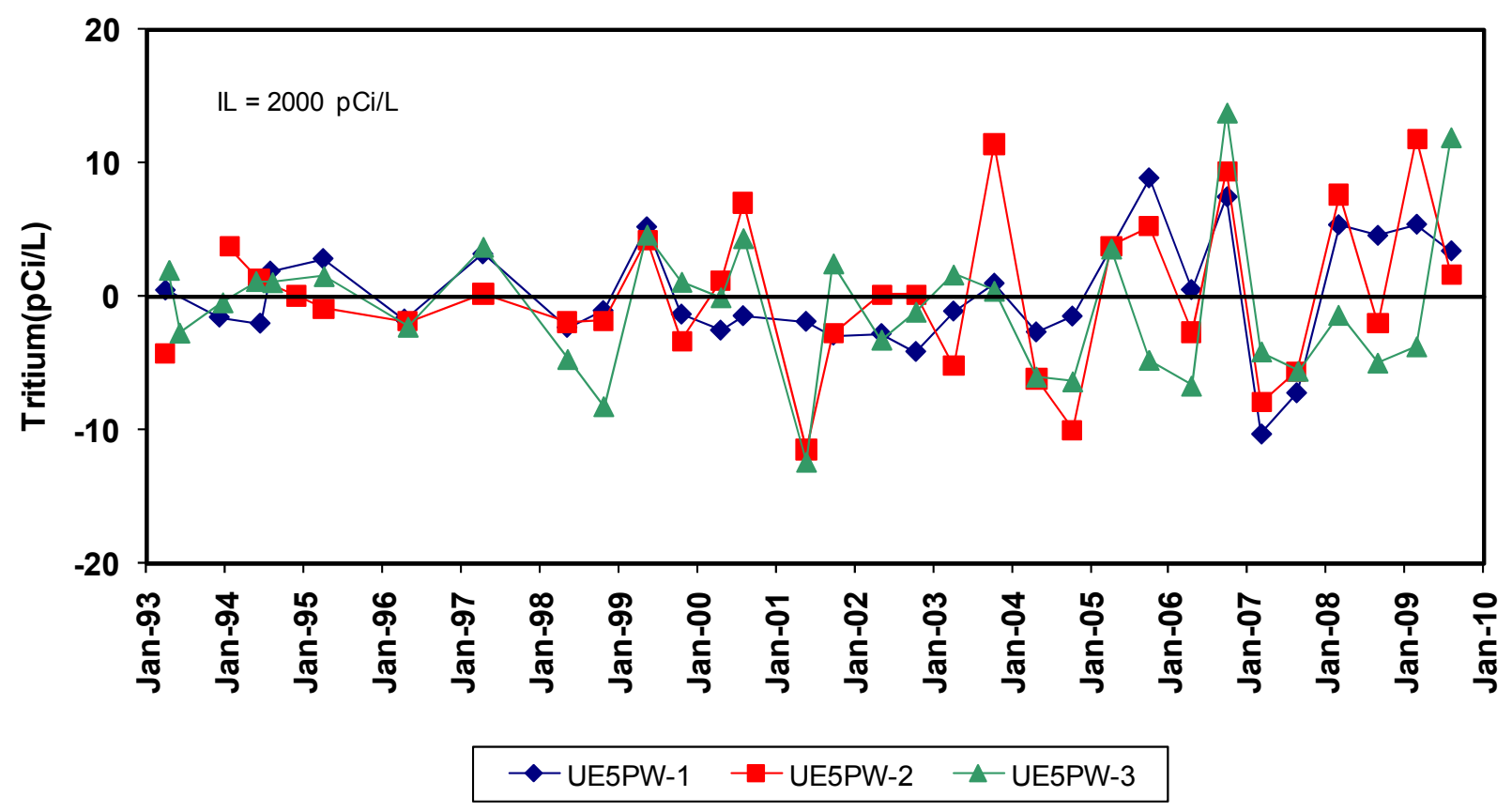

Figure 2-5 Area 5 RWMS Time Series Plot of Tritium

\subsubsection{General Water Chemistry Parameters}

General water chemistry analyses during 2008 for cations ( $\mathrm{Ca}, \mathrm{Mg}, \mathrm{Na}, \mathrm{K}, \mathrm{Fe})$, anions $(\mathrm{Cl}, \mathrm{F}$, $\mathrm{SO}_{4}, \mathrm{HCO}_{3}$ ), and $\mathrm{SiO}_{2}$ indicate similar groundwater in all three wells and no changes in groundwater chemistry (Table 2-7, Table 2-8, and Table 2-9). Stiff plots for 2008 and 2009 indicate similar groundwater chemistry for all three wells and no changes in the groundwater chemistry (Figure 2-6). A piper diagram for the same water chemistry data from 2006 through 2009 indicates that the groundwater is a sodium-bicarbonate type (Figure 2-7).

Groundwater temperatures measured in March 2009 ranged from $17.5^{\circ} \mathrm{C}$ to $17.8^{\circ} \mathrm{C}\left(63.5^{\circ} \mathrm{F}\right.$ to $\left.64.0^{\circ} \mathrm{F}\right)$ and in August 2009 ranged from $21.7^{\circ} \mathrm{C}$ to $22.6^{\circ} \mathrm{C}\left(71.0^{\circ} \mathrm{F}\right.$ to $\left.72.7^{\circ} \mathrm{F}\right)$. Temperature measurements are collected at the ground surface and are influenced by the ambient air temperature. 
Table 2-7 UE5PW-1 General Water Chemistry Values in mg/L

\begin{tabular}{|c|c|c|c|c|c|c|c|c|c|c|c|}
\hline Date & $\mathrm{Ca}$ & Mg & $\mathbf{K}$ & $\mathrm{Na}$ & Mn & $\mathrm{Fe}$ & $\mathrm{SiO}_{2}$ & $\mathrm{SO}_{4}$ & $\mathrm{HCO}_{3}$ & $\mathrm{Cl}$ & $\mathbf{F}$ \\
\hline $3 / 31 / 1993$ & No analysis & No analysis & No analysis & 48 & $<0.006$ & 0.013 & No analysis & 32 & 167 & 9.2 & 1.2 \\
\hline 6/6/1993 & No analysis & No analysis & No analysis & 58 & $<0.001$ & 0.059 & No analysis & 37 & 161 & 9.7 & 1.4 \\
\hline 9/1/1993 & No analysis & No analysis & No analysis & 56 & 0.0066 & 0.027 & No analysis & No analysis & 158 & 8.4 & 5.7 \\
\hline 12/7/1993 & No analysis & No analysis & No analysis & 57 & $<0.0012$ & 0.012 & No analysis & 36 & 150 & 9.9 & 1.5 \\
\hline 6/15/1994 & No analysis & No analysis & No analysis & 61 & $<0.004$ & 0.01 & No analysis & No analysis & No analysis & No analysis & No analysis \\
\hline 8/1/1994 & No analysis & No analysis & No analysis & 53 & $<0.0012$ & 0.021 & No analysis & 36 & No analysis & 10.0 & No analysis \\
\hline 4/4/1995 & No analysis & No analysis & No analysis & 58 & $<0.01$ & $<0.05$ & No analysis & 34 & No analysis & 9.9 & No analysis \\
\hline 4/16/1996 & No analysis & No analysis & No analysis & 61 & $<0.001$ & 0.02 & No analysis & 34 & No analysis & 9.9 & No analysis \\
\hline $11 / 5 / 1997$ & 15.5 & 5.6 & 6.4 & 57.8 & No analysis & 0.012 & No analysis & 35.2 & 151 & 10.2 & 1.2 \\
\hline $5 / 13 / 1998$ & 14.0 & 5.4 & 5.2 & 55.8 & 0.0015 & 0.034 & 54.2 & 34.6 & 151 & 9.6 & 1.1 \\
\hline 10/28/1998 & 14.9 & 5.6 & 6.9 & 57.6 & 0.0015 & 0.024 & 60.5 & 34.0 & 160 & 9.7 & 1.1 \\
\hline $5 / 19 / 1999$ & 12.5 & 5.3 & 6.9 & 61.0 & $<0.0025$ & $<0.05$ & 68.5 & 34.0 & 146 & 10.0 & 1.0 \\
\hline 10/27/1999 & 14.5 & 6.0 & 6.6 & 63.5 & $<0.008$ & $<0.009$ & 62.0 & 35.0 & 159 & 8.8 & 1.1 \\
\hline $4 / 26 / 2000$ & 12.8 & 4.8 & 6.7 & 53.7 & 0.001 & 0.0326 & 58.4 & 35.7 & 165 & 10.0 & 1.0 \\
\hline $8 / 9 / 2000$ & 15.0 & 4.9 & 6.6 & 52.0 & 0.00045 & $<0.0164$ & 59.9 & 37.1 & 146 & 10.4 & 1.1 \\
\hline $5 / 29 / 2001$ & 14.4 & 4.9 & 6.0 & 59.0 & $<0.025$ & 0.01215 & 61.7 & No analysis & 143 & No analysis & No analysis \\
\hline $10 / 3 / 2001$ & 13.7 & 4.8 & 6.7 & 51.0 & 0.00020 & $<0.0156$ & 58.3 & 36.0 & 151 & 10.2 & 1.0 \\
\hline $10 / 22 / 2002$ & 14.6 & 5.2 & 6.4 & 50.0 & 0.0002 & 0.0181 & 60.7 & 35.6 & 143 & 10.1 & 1.0 \\
\hline 4/15/2003 & 13.7 & 5.0 & 6.2 & 58.0 & $<0.0005$ & 0.011 & 59.2 & 32.9 & 150 & 12.3 & 1.0 \\
\hline $10 / 22 / 2003$ & 14.0 & 5.0 & 6.0 & 58.1 & $<0.0016$ & 0.0141 & 61.2 & 36.6 & No analysis & 9.5 & 1.1 \\
\hline $5 / 4 / 2004$ & 12.9 & 4.6 & 6.4 & 55.3 & 0.0027 & 0.0374 & 54.4 & 34.4 & 154 & 9.8 & 1.1 \\
\hline $10 / 19 / 2004$ & 13.1 & 5.2 & 6.0 & 56.2 & $<0.0003$ & 0.0279 & 59.9 & 37.3 & 168 & 10.1 & 1.0 \\
\hline $4 / 19 / 2005$ & 13.8 & 4.8 & 6.6 & 55.1 & $<0.0006$ & 0.007 & 58.6 & 39.6 & 149 & 10.5 & 1.0 \\
\hline $10 / 11 / 2005$ & 13.4 & 5.0 & 6.1 & 50.5 & $<0.0002$ & $<0.026$ & 61.2 & 35.7 & 156 & 9.7 & 1.0 \\
\hline $4 / 26 / 2006$ & 14.6 & 5.3 & 6.3 & 60.4 & $<0.0032$ & $<0.0054$ & 63.3 & 35.4 & 149 & 10.7 & 1.2 \\
\hline $10 / 10 / 2006$ & 14.0 & 5.2 & 5.9 & 58.8 & 0.0007 & $<0.0048$ & 61.4 & 33.8 & 148 & 9.9 & 0.9 \\
\hline $3 / 19 / 2007$ & 15.7 & 5.4 & 6.0 & 57.4 & $<0.0036$ & 0.0124 & 64.0 & 37.7 & 151 & 10.5 & 1.0 \\
\hline $8 / 29 / 2007$ & 15.4 & 5.4 & 6.2 & 59.0 & 0.00046 & 0.0058 & 64.6 & 35.9 & 148 & 10.0 & 1.2 \\
\hline $3 / 11 / 2008$ & 14.0 & 5.4 & 6.3 & 60.4 & $<0.00045$ & 0.0066 & 63.1 & 37.4 & 149 & 11.1 & 1.2 \\
\hline $9 / 10 / 2008$ & 14.3 & 5.5 & 6.4 & 59.1 & $<0.0009$ & $<0.045$ & 62.5 & 34.7 & 155 & 11.0 & 1.2 \\
\hline $3 / 10 / 2009$ & 13.4 & 5.3 & 6.1 & 58.1 & $<0.005$ & $<0.100$ & 58.6 & 35.7 & 174 & 10.1 & 1.1 \\
\hline $8 / 18 / 2009$ & 13.4 & 5.3 & 6.0 & 58.3 & 0.00113 & 0.0168 & 61.8 & 37.1 & 160 & 11.0 & 1.0 \\
\hline
\end{tabular}

Data source: Data before 10/27/1999 from BN, 2001 
Table 2-8 UE5PW-2 General Water Chemistry Values in mg/L

\begin{tabular}{|c|c|c|c|c|c|c|c|c|c|c|c|}
\hline Date & $\mathrm{Ca}$ & Mg & $\mathbf{K}$ & $\mathrm{Na}$ & Mn & $\mathrm{Fe}$ & $\mathrm{SiO}_{2}$ & $\mathrm{SO}_{4}$ & $\mathrm{HCO}_{3}$ & $\mathrm{Cl}$ & $\mathbf{F}$ \\
\hline $3 / 24 / 1993$ & No analysis & No analysis & No analysis & 46 & 0.11 & 0.062 & No analysis & 28 & 159 & 8.4 & 1.0 \\
\hline $6 / 22 / 1993$ & No analysis & No analysis & No analysis & 54 & 0.032 & 0.25 & No analysis & 30 & 183 & 9.7 & 1.1 \\
\hline $11 / 15 / 1993$ & No analysis & No analysis & No analysis & 51 & $<0.004$ & 0.180 & No analysis & 31 & 171 & 9.4 & 1.3 \\
\hline $1 / 19 / 1994$ & No analysis & No analysis & No analysis & 45 & $<0.0012$ & 0.074 & No analysis & 29 & 159 & No analysis & 1.2 \\
\hline 6/7/1994 & No analysis & No analysis & No analysis & 55 & $<0.004$ & 0.14 & No analysis & No analysis & No analysis & No analysis & No analysis \\
\hline 11/29/1994 & No analysis & No analysis & No analysis & No analysis & No analysis & No analysis & No analysis & 28 & No analysis & 8.0 & No analysis \\
\hline 4/4/1995 & No analysis & No analysis & No analysis & 50 & $<0.01$ & $<0.05$ & No analysis & 28 & No analysis & 8.5 & No analysis \\
\hline 4/30/1996 & No analysis & No analysis & No analysis & 51 & $<0.001$ & 0.0127 & No analysis & 29 & No analysis & 8.3 & No analysis \\
\hline $11 / 5 / 1997$ & 17.4 & 6.8 & 4.9 & 50.6 & No analysis & 0.018 & No analysis & 28.9 & 140 & 8.6 & 0.9 \\
\hline $5 / 13 / 1998$ & 14.8 & 5.7 & 3.8 & 45.2 & $<0.0011$ & 0.073 & 50.8 & 28.2 & 151 & 8.1 & 1.0 \\
\hline 10/28/1998 & 15.8 & 6.2 & 5.6 & 47.4 & 0.0009 & 0.018 & 55.9 & 28.4 & 157 & 8.3 & 1.0 \\
\hline $5 / 19 / 1999$ & 15.0 & 6.3 & 6.2 & 52.0 & $<0.0025$ & $<0.05$ & 62.0 & 27.5 & 134 & 8.7 & 0.9 \\
\hline 10/27/1999 & 16.0 & 6.7 & 5.7 & 52.0 & $<0.0008$ & $<0.009$ & 55.6 & 28.0 & 152 & 7.4 & 1.0 \\
\hline $4 / 26 / 2000$ & 15.3 & 6.5 & 5.6 & 45.6 & 0.0007 & 0.0288 & 55.8 & 29.1 & 177 & 8.6 & 0.8 \\
\hline $8 / 9 / 2000$ & 17.0 & 6.6 & 5.3 & 44.5 & $<0.0002$ & $<0.0164$ & 59.2 & 28.8 & 155 & 9.3 & 0.9 \\
\hline $5 / 29 / 2001$ & 16.6 & 6.6 & 4.8 & 48.8 & $<0.0088$ & $<0.0107$ & 60.4 & No analysis & 152 & No analysis & No analysis \\
\hline $10 / 3 / 2001$ & 16.0 & 6.7 & 5.5 & 44.7 & 0.00017 & 0.0214 & 58.8 & 28.4 & 152 & 8.7 & 1.0 \\
\hline $10 / 22 / 2002$ & 17.6 & 7.1 & 5.3 & 44.4 & 0.0031 & $<0.0181$ & 63.0 & 28.7 & 149 & 8.7 & 0.8 \\
\hline $4 / 15 / 2003$ & 16.3 & 6.6 & 5.3 & 50.8 & $<0.0005$ & $<0.0101$ & 60.3 & 26.7 & 157 & 9.8 & 0.8 \\
\hline $10 / 22 / 2003$ & 16.1 & 6.6 & 5.2 & 49.6 & $<0.0016$ & 0.0618 & 60.5 & 29.5 & 141 & 8.8 & 0.9 \\
\hline $5 / 4 / 2004$ & 16.0 & 6.3 & 5.4 & 47.2 & $<0.0007$ & 0.0397 & 58.2 & 28.1 & 159 & 8.2 & 0.9 \\
\hline $10 / 19 / 2004$ & 15.7 & 6.7 & 5.1 & 48.6 & $<0.0003$ & $<0.0279$ & 59.7 & 29.6 & 169 & 8.9 & 0.9 \\
\hline $4 / 19 / 2005$ & 16.3 & 6.3 & 5.2 & 44.9 & $<0.0006$ & 0.0115 & 58.6 & 31.3 & 133 & 8.4 & 0.9 \\
\hline $10 / 11 / 2005$ & 16.0 & 6.8 & 5.0 & 44.0 & $<0.0002$ & 0.0270 & 62.2 & 29.0 & 167 & 8.1 & 0.9 \\
\hline $4 / 26 / 2006$ & 16.6 & 6.7 & 5.4 & 51.2 & $<0.0032$ & 0.0612 & 62.5 & 28.1 & 152 & 8.8 & 1.1 \\
\hline $10 / 10 / 2006$ & 16.5 & 6.5 & 5.2 & 48.0 & $<0.0007$ & 0.0170 & 61.2 & 27.2 & 156 & 8.6 & 1.1 \\
\hline $3 / 19 / 2007$ & 16.8 & 6.6 & 5.4 & 49.8 & $<0.0036$ & 0.0387 & 62.9 & 42.2 & 149 & 11.3 & 0.9 \\
\hline $8 / 29 / 2007$ & 16.9 & 6.7 & 5.2 & 50.5 & $<0.00045$ & 0.0098 & 63.7 & 27.9 & 151 & 9.0 & 1.1 \\
\hline $3 / 11 / 2008$ & 16.7 & 6.7 & 5.2 & 50.5 & $<0.00045$ & 0.0159 & 60.3 & 30.7 & 149 & 10.0 & 1.0 \\
\hline $9 / 10 / 2008$ & 16.8 & 7.0 & 5.7 & 52.7 & 0.0020 & $<0.045$ & 60.3 & 28.7 & 152 & 9.2 & 1.0 \\
\hline $3 / 10 / 2009$ & 15.9 & 6.7 & 5.0 & 50.0 & $<0.005$ & $<0.100$ & 61.6 & 28.9 & 165 & 8.4 & 1.0 \\
\hline $8 / 18 / 2009$ & 15.5 & 6.8 & 5.1 & 50.9 & 0.00066 & 0.0123 & 61.2 & 29.1 & 155 & 8.8 & 0.9 \\
\hline
\end{tabular}

Data source: Data before 10/27/1999 from BN, 2001 
Table 2-9 UE5PW-3 General Water Chemistry Values in mg/L

\begin{tabular}{|c|c|c|c|c|c|c|c|c|c|c|c|}
\hline Date & $\mathrm{Ca}$ & Mg & $\mathbf{K}$ & $\mathrm{Na}$ & Mn & $\mathrm{Fe}$ & $\mathrm{SiO}_{2}$ & $\mathrm{SO}_{4}$ & $\mathrm{HCO}_{3}$ & $\mathrm{Cl}$ & $\mathbf{F}$ \\
\hline $04 / 14 / 93$ & No analysis & No analysis & No analysis & 46 & 0.042 & 0.024 & No analysis & 31 & 157 & 8.5 & 1.3 \\
\hline $06 / 02 / 93$ & No analysis & No analysis & No analysis & 53 & 0.009 & 0.014 & No analysis & 31 & 162 & 9.1 & 1.2 \\
\hline $10 / 12 / 93$ & No analysis & No analysis & No analysis & 57 & $<0.006$ & 0.11 & No analysis & 30 & 156 & 7.9 & 1.2 \\
\hline $12 / 20 / 93$ & No analysis & No analysis & No analysis & 48 & $<0.0012$ & 0.1 & No analysis & 33 & 156 & 8.7 & 1.3 \\
\hline $05 / 24 / 94$ & No analysis & No analysis & No analysis & 56 & $<0.0012$ & 0.02 & No analysis & No analysis & No analysis & No analysis & No analysis \\
\hline 08/08/94 & No analysis & No analysis & No analysis & 51 & $<0.0012$ & $<0.009$ & No analysis & 33 & No analysis & 8.9 & No analysis \\
\hline $04 / 05 / 95$ & No analysis & No analysis & No analysis & 55 & $<0.01$ & $<0.05$ & No analysis & 31 & No analysis & 8.8 & No analysis \\
\hline $04 / 30 / 96$ & No analysis & No analysis & No analysis & 57 & $<0.001$ & 0.0088 & No analysis & 32 & No analysis & 8.7 & No analysis \\
\hline $11 / 05 / 97$ & 16.8 & 6.1 & 4.3 & 55.5 & No analysis & 0.0133 & No analysis & 32.1 & 140 & 9.2 & 1.1 \\
\hline $05 / 13 / 98$ & 15.8 & 5.8 & 3.3 & 53.8 & $<0.0011$ & 0.025 & 56.6 & 30.9 & 151 & 8.4 & 1.0 \\
\hline $10 / 28 / 98$ & 15.6 & 5.7 & 4.2 & 53.7 & 0.0009 & 0.0154 & 57.1 & 31.8 & 156 & 8.7 & 1.0 \\
\hline $05 / 19 / 99$ & 15.0 & 5.8 & 4.8 & 56.0 & $<0.0025$ & $<0.05$ & 66.3 & 30.5 & 146 & 9.2 & 0.9 \\
\hline $10 / 27 / 99$ & 16.0 & 6.4 & 5.0 & 58.5 & $<0.0008$ & $<0.009$ & 59.9 & 31.0 & 159 & 7.7 & 0.9 \\
\hline $04 / 26 / 00$ & 15.3 & 5.9 & 4.5 & 49.8 & 0.00033 & 0.0178 & 58.5 & 32.0 & 169 & 9.1 & 0.9 \\
\hline 08/09/00 & 16.0 & 5.8 & 4.3 & 48.3 & $<0.0002$ & $<0.0164$ & 57.8 & 32.6 & 162 & 9.9 & 1.0 \\
\hline 05/29/01 & 16.4 & 5.9 & 4.0 & 54.8 & 0.00180 & $<0.0107$ & 60.5 & No analysis & 151 & No analysis & No analysis \\
\hline $10 / 03 / 01$ & 15.6 & 6.0 & 4.5 & 48.4 & 0.00022 & 0.0237 & 57.9 & 31.5 & 154 & 8.9 & 1.0 \\
\hline $10 / 22 / 02$ & 17.2 & 6.2 & 4.3 & 47.6 & $<0.0002$ & $<0.0181$ & 60.5 & 32.2 & 143 & 9.3 & 0.9 \\
\hline $04 / 15 / 03$ & 16.0 & 5.9 & 4.5 & 54.7 & 0.00083 & 0.0195 & 58.4 & 29.3 & 144 & 11.8 & 0.8 \\
\hline $10 / 21 / 03$ & 16.3 & 5.8 & 4.1 & 54.4 & $<0.0016$ & 0.0212 & 59.5 & 32.5 & 160 & 9.2 & 1.0 \\
\hline $05 / 04 / 04$ & 16.1 & 5.6 & 4.7 & 52.2 & 0.0019 & 0.0453 & 58.2 & 31.1 & 155 & 8.7 & 1.0 \\
\hline $10 / 20 / 04$ & 15.6 & 5.9 & 4.0 & 52.3 & $<0.0003$ & $<0.0279$ & 58.4 & 32.0 & 166 & 9.4 & 0.8 \\
\hline $04 / 19 / 05$ & 16.2 & 5.6 & 4.5 & 50.9 & $<0.0006$ & 0.0319 & 57.8 & 34.4 & 148 & 8.8 & 0.9 \\
\hline $10 / 11 / 05$ & 16.1 & 6.1 & 4.3 & 48.5 & $<0.0002$ & $<0.026$ & 61.4 & 32.5 & 156 & 8.5 & 0.9 \\
\hline $04 / 26 / 06$ & 16.6 & 6.1 & 4.2 & 58.1 & $<0.0032$ & 0.0057 & 61.6 & 31.6 & 159 & 9.4 & 1.2 \\
\hline $10 / 10 / 06$ & 15.9 & 5.5 & 4.0 & 49.7 & 0.0007 & 0.0114 & 57.3 & 30.1 & 152 & 9.0 & 1.0 \\
\hline 03/19/07 & 16.8 & 6.1 & 4.0 & 55.5 & $<0.0036$ & 0.0921 & 61.2 & 19.9 & 149 & 9.3 & 0.8 \\
\hline $09 / 05 / 07$ & 16.5 & 5.9 & 4.3 & 54.7 & 0.0012 & 0.0041 & 60.1 & 32.5 & 149 & 9.8 & 1.1 \\
\hline $03 / 11 / 08$ & 16.7 & 6.1 & 4.2 & 57.2 & $<0.00045$ & 0.0045 & 58.8 & 32.1 & 144 & 9.9 & 1.0 \\
\hline $09 / 10 / 08$ & 16.4 & 6.1 & 4.5 & 56.4 & $<0.0009$ & $<0.045$ & 58.8 & 35.9 & 165 & 9.5 & 1.0 \\
\hline 03/10/09 & 15.9 & 6.0 & 4.2 & 55.6 & $<0.005$ & 0.100 & 59.0 & 31.7 & 155 & 9.0 & 0.9 \\
\hline 08/18/09 & 15.4 & 5.9 & 4.0 & 54.7 & 0.00062 & 0.0112 & 58.4 & 32.5 & 152 & 9.4 & 0.9 \\
\hline
\end{tabular}

Data source: Data before 10/27/1999 from BN, 2001 
03/11/2008

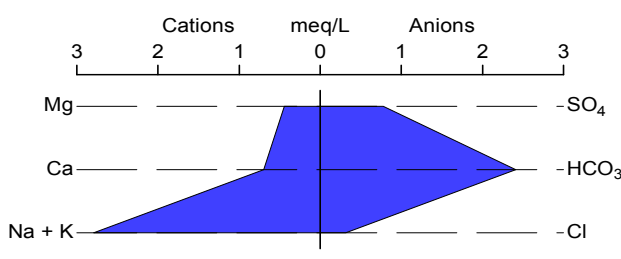

09/10/2008

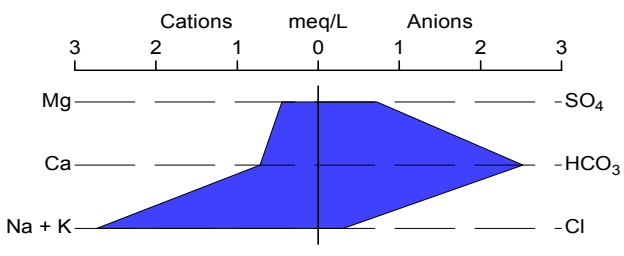

03/10/2009

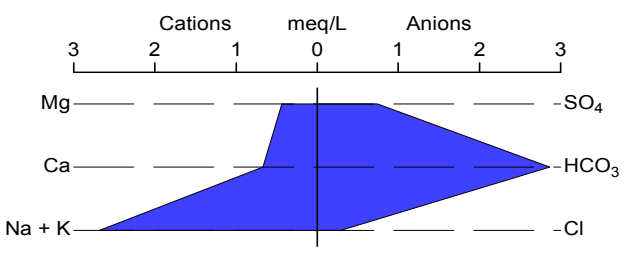

$08 / 18 / 2009$

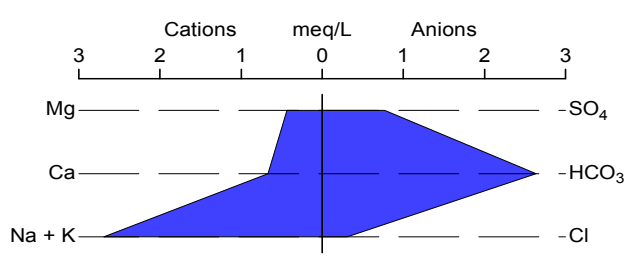

$03 / 11 / 2008$

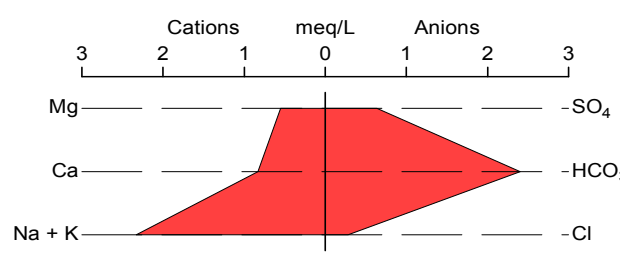

09/10/2008

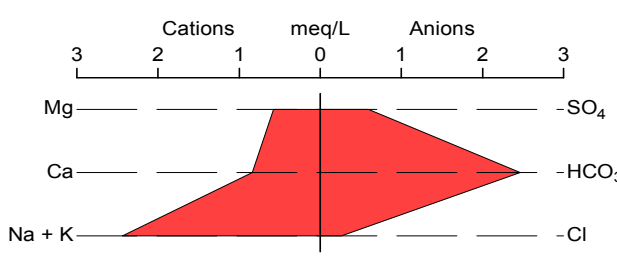

03/10/2009

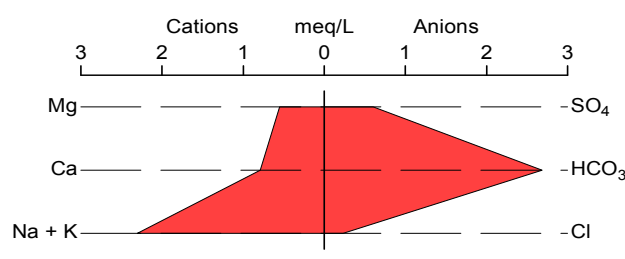

08/18/2009

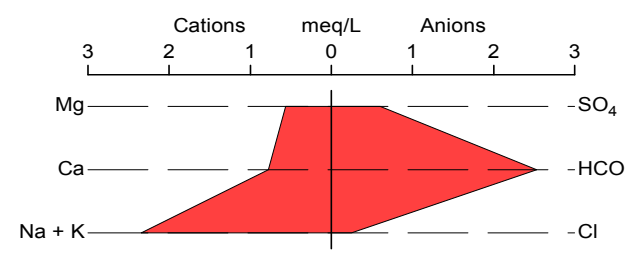

$03 / 11 / 2008$

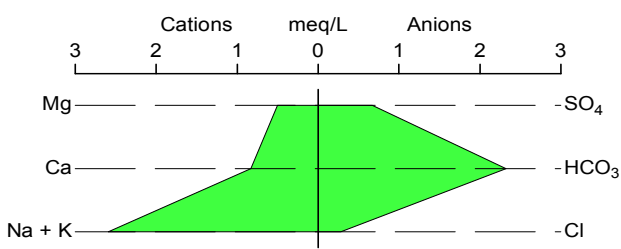

09/10/2008

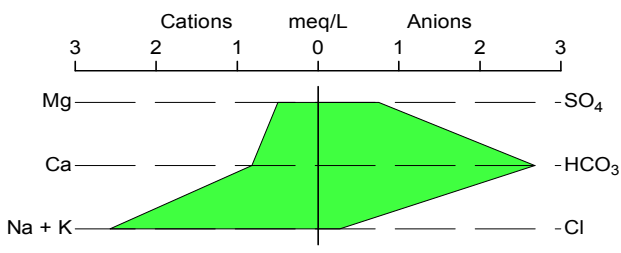

03/10/2009

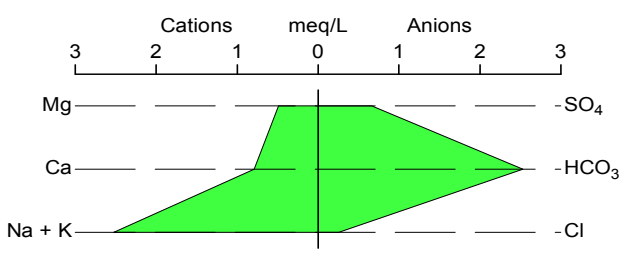

$08 / 18 / 2009$

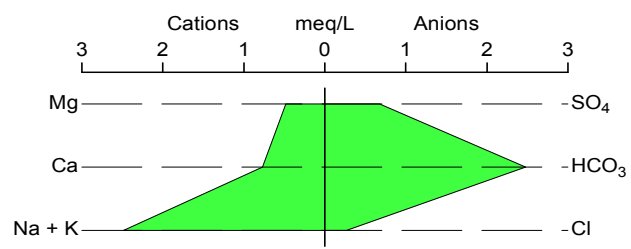

\section{\begin{tabular}{lll}
\hline UE5PW-1 \\
UE5PW-2 $\square$ UE5PW-3
\end{tabular}}

Figure 2-6 Area 5 RWMS Stiff Diagrams for 2008 and 2009 

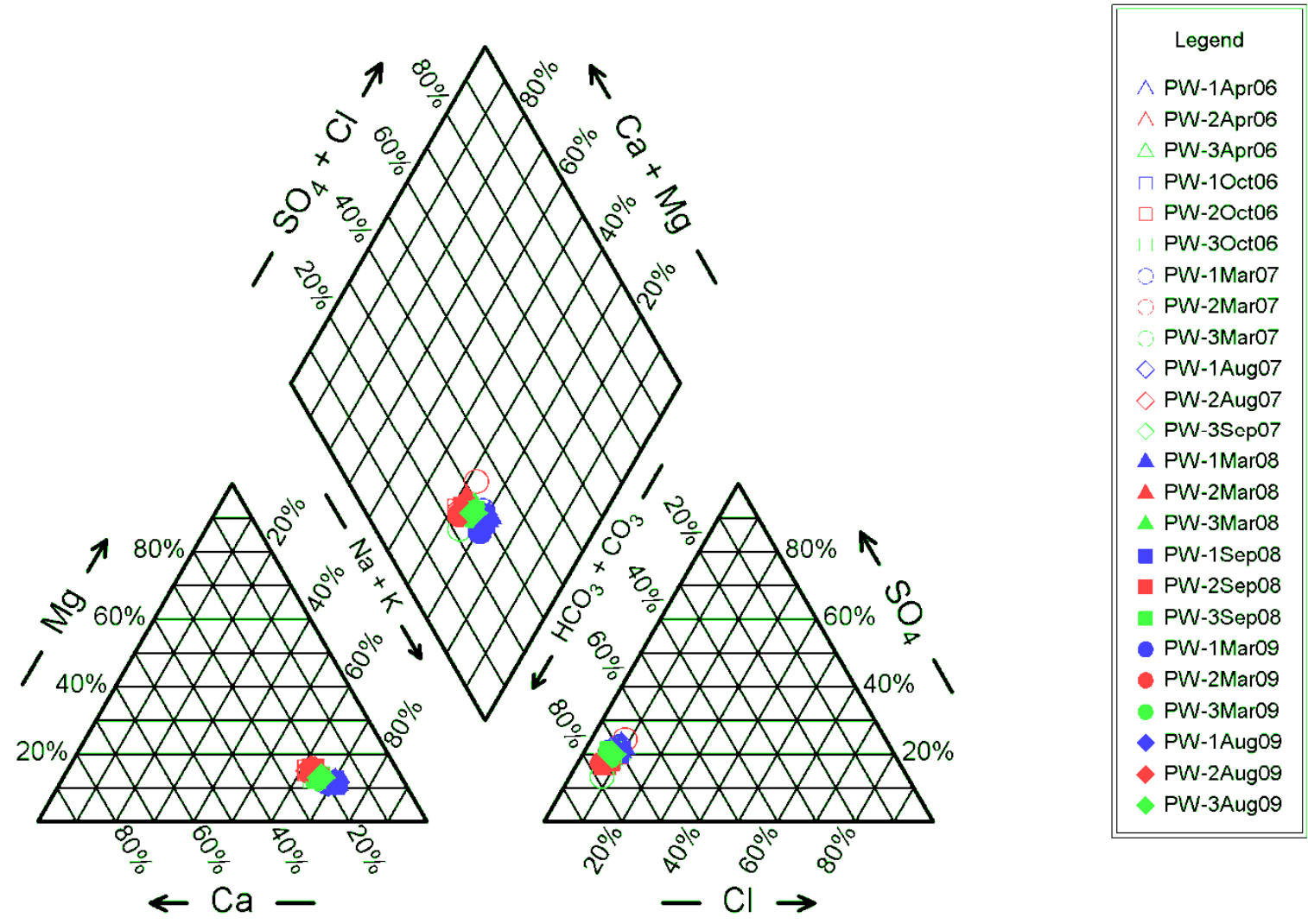

Figure 2-7 Area 5 RWMS Four-Year Piper Diagram

\subsubsection{Groundwater Elevation}

Groundwater elevations in UE5PW-1, UE5PW-2, and UE5PW-3 are measured quarterly using an electronic water-level tape (Table 2-10; Figure 2-8). The 2009 average depths to water from top of casing are $235.79 \mathrm{~m}$ (773.59 ft), $256.42 \mathrm{~m}(841.26 \mathrm{ft})$, and $271.53 \mathrm{~m}(890.83 \mathrm{ft})$ for UE5PW-1, UE5PW-2, and UE5PW-3, respectively. These measurements are corrected for borehole deviation (REECo, 1994).

The 2009 average groundwater elevations are $733.71 \mathrm{~m}(2,407.17 \mathrm{ft}) \mathrm{AMSL}, 733.70 \mathrm{~m}$ $(2,407.14 \mathrm{ft}) \mathrm{AMSL}$, and $733.70 \mathrm{~m}(2,407.14 \mathrm{ft})$ AMSL for UE5PW-1, UE5PW-2, and UE5PW-3, respectively. These measurements are corrected for borehole deviation (REECo, 1994). Based on the similar groundwater elevations, the groundwater table is essentially flat with little or no flow. Groundwater gradient, velocity, and flow direction are calculated from the groundwater elevations, borehole locations, and aquifer hydraulic properties (Table 2-11; Appendix B). The average calculated flow velocity during 2009 was 0.08 meters/year and the flow direction was south. The very low calculated flow velocities and the fluctuating flow directions indicate little or no groundwater flow. 
Table 2-10 Area 5 RWMS Groundwater Elevation Data

\begin{tabular}{|c|c|c|c|c|c|c|}
\hline $\begin{array}{c}\text { Well } \\
\text { Characteristics }^{\mathrm{a}}\end{array}$ & \multicolumn{2}{|c|}{ UE5PW-1 } & \multicolumn{2}{|c|}{ UE5PW-2 } & \multicolumn{2}{|c|}{ UE5PW-3 } \\
\hline Northing $^{\mathrm{b}}(\mathrm{m})$ & \multicolumn{2}{|c|}{$233,386.48$} & \multicolumn{2}{|c|}{$234,817.13$} & \multicolumn{2}{|c|}{$235,089.93$} \\
\hline Easting $^{\mathrm{b}}(\mathrm{m})$ & \multicolumn{2}{|c|}{$216,357.08$} & \multicolumn{2}{|c|}{$216,376.00$} & \multicolumn{2}{|c|}{$214,415.04$} \\
\hline $\begin{array}{l}\text { Well Casing } \\
\text { Elevation }^{c}(\mathrm{~m})\end{array}$ & \multicolumn{2}{|c|}{969.37} & \multicolumn{2}{|c|}{990.12} & \multicolumn{2}{|c|}{$1,005.22$} \\
\hline $\begin{array}{l}\text { Casing Stickup } \\
\text { Height }^{d}(m)\end{array}$ & \multicolumn{2}{|c|}{0.72} & \multicolumn{2}{|c|}{0.68} & \multicolumn{2}{|c|}{0.76} \\
\hline $\begin{array}{l}\text { Land Surface } \\
\text { Elevation }(\mathrm{m})\end{array}$ & \multicolumn{2}{|c|}{968.73} & \multicolumn{2}{|c|}{989.54} & \multicolumn{2}{|c|}{$1,004.50$} \\
\hline $\begin{array}{l}\text { Borehole Deviation } \\
\text { Correction (m) }\end{array}$ & \multicolumn{2}{|c|}{0.08} & \multicolumn{2}{|c|}{0.21} & \multicolumn{2}{|c|}{0.02} \\
\hline Date & $\begin{array}{c}\text { Depth to } \\
\text { Water } \\
\text { (m below } \\
\text { Top of } \\
\text { Casing) }\end{array}$ & $\begin{array}{c}\text { Water } \\
\text { Table } \\
\text { Elevation } \\
\text { (m) }\end{array}$ & $\begin{array}{l}\text { Depth to } \\
\text { Water } \\
\text { (m below } \\
\text { Top of } \\
\text { Casing) }\end{array}$ & $\begin{array}{c}\text { Water } \\
\text { Table } \\
\text { Elevation } \\
\text { (m) }\end{array}$ & $\begin{array}{l}\text { Depth to } \\
\text { Water } \\
\text { ( } \mathrm{m} \text { below } \\
\text { Top of } \\
\text { Casing) }\end{array}$ & $\begin{array}{c}\text { Water } \\
\text { Table } \\
\text { Elevation } \\
\quad(\mathrm{m})\end{array}$ \\
\hline $03 / 22 / 1993$ & 235.55 & 733.82 & 256.38 & 733.74 & 271.69 & 733.53 \\
\hline 03/23/1993 & 235.53 & 733.84 & 256.48 & 733.64 & 271.68 & 733.54 \\
\hline 03/24/1993 & 235.53 & 733.84 & 256.36 & 733.76 & 271.69 & 733.53 \\
\hline 03/25/1993 & 235.53 & 733.84 & 256.35 & 733.77 & 271.69 & 733.53 \\
\hline 03/29/1993 & 235.59 & 733.78 & 256.38 & 733.74 & 271.73 & 733.49 \\
\hline 03/30/1993 & 235.62 & 733.75 & 256.43 & 733.69 & 271.75 & 733.47 \\
\hline 03/31/1993 & 235.62 & 733.75 & 256.44 & 733.68 & 271.74 & 733.48 \\
\hline 04/01/1993 & 235.54 & 733.83 & 256.37 & 733.75 & 271.69 & 733.53 \\
\hline 04/05/1993 & 235.51 & 733.86 & 256.35 & 733.77 & 271.67 & 733.55 \\
\hline 04/06/1993 & 235.59 & 733.78 & 256.40 & 733.72 & 271.75 & 733.47 \\
\hline 05/10/1993 & 235.64 & 733.73 & 256.46 & 733.66 & 271.76 & 733.46 \\
\hline 05/11/1993 & 235.56 & 733.81 & 256.42 & 733.70 & 271.70 & 733.52 \\
\hline 05/12/1993 & 235.54 & 733.83 & 256.40 & 733.72 & 271.72 & 733.50 \\
\hline 05/13/1993 & 235.61 & 733.76 & 256.45 & 733.67 & 271.75 & 733.47 \\
\hline 05/17/1993 & 235.61 & 733.76 & 256.45 & 733.67 & 271.74 & 733.48 \\
\hline 05/18/1993 & 235.59 & 733.78 & 256.45 & 733.67 & 271.74 & 733.48 \\
\hline 05/19/1993 & 235.59 & 733.78 & 256.44 & 733.68 & 271.73 & 733.49 \\
\hline 05/20/1993 & 235.54 & 733.83 & 256.39 & 733.73 & 271.70 & 733.52 \\
\hline 05/24/1993 & 235.60 & 733.77 & 256.43 & 733.69 & 271.74 & 733.48 \\
\hline 05/25/1993 & 235.61 & 733.76 & 256.45 & 733.67 & 271.74 & 733.48 \\
\hline 06/01/1993 & 235.58 & 733.79 & 256.43 & 733.69 & 271.73 & 733.49 \\
\hline 06/07/1993 & 235.64 & 733.73 & 256.46 & 733.66 & 271.76 & 733.46 \\
\hline 06/14/1993 & 235.61 & 733.76 & 256.46 & 733.66 & 271.74 & 733.48 \\
\hline 06/21/1993 & 235.58 & 733.79 & 256.43 & 733.69 & 271.73 & 733.49 \\
\hline 07/26/1993 & 235.59 & 733.78 & 256.45 & 733.67 & 271.74 & 733.48 \\
\hline 08/03/1993 & 235.54 & 733.83 & 256.42 & 733.70 & 271.70 & 733.52 \\
\hline 08/09/1993 & 235.62 & 733.75 & 256.46 & 733.66 & 271.75 & 733.47 \\
\hline 08/16/1993 & 235.59 & 733.78 & 256.42 & 733.70 & 271.73 & 733.49 \\
\hline 08/30/1993 & 235.58 & 733.79 & 256.43 & 733.69 & 271.72 & 733.50 \\
\hline $12 / 28 / 1993$ & 235.59 & 733.78 & 256.47 & 733.65 & 271.74 & 733.48 \\
\hline $01 / 03 / 1994$ & 235.57 & 733.80 & 256.44 & 733.68 & 271.70 & 733.52 \\
\hline 02/02/1994 & 235.53 & 733.84 & 256.44 & 733.68 & 271.66 & 733.56 \\
\hline 02/22/1994 & 235.60 & 733.77 & 256.43 & 733.69 & 271.71 & 733.51 \\
\hline
\end{tabular}




\begin{tabular}{|c|c|c|c|c|c|c|}
\hline 02/28/1994 & 235.60 & 733.77 & 256.45 & 733.67 & 271.70 & 733.52 \\
\hline 03/07/1994 & 235.54 & 733.83 & 256.38 & 733.74 & 271.66 & 733.56 \\
\hline 03/14/1994 & 235.55 & 733.82 & 256.45 & 733.67 & 271.67 & 733.55 \\
\hline 03/21/1994 & 235.56 & 733.81 & 256.38 & 733.74 & 271.68 & 733.54 \\
\hline 03/28/1994 & 235.63 & 733.74 & 256.47 & 733.65 & 271.70 & 733.52 \\
\hline 04/04/1994 & 235.53 & 733.84 & 256.40 & 733.72 & 271.66 & 733.56 \\
\hline 04/13/1994 & 235.55 & 733.82 & 256.43 & 733.69 & 271.65 & 733.57 \\
\hline 04/20/1994 & 235.51 & 733.86 & 256.38 & 733.74 & 271.64 & 733.58 \\
\hline 04/26/1994 & 235.55 & 733.82 & 256.35 & 733.77 & 271.65 & 733.57 \\
\hline 01/18/1995 & 235.63 & 733.74 & 256.45 & 733.67 & 271.62 & 733.60 \\
\hline 04/03/1995 & 235.57 & 733.80 & 256.39 & 733.73 & 271.61 & 733.61 \\
\hline 01/16/1996 & 235.36 & 734.01 & 256.13 & 733.99 & 271.35 & 733.87 \\
\hline 04/15/1996 & 235.56 & 733.81 & 256.30 & 733.82 & 271.43 & 733.79 \\
\hline 10/01/1996 & 235.54 & 733.83 & 256.32 & 733.80 & 271.51 & 733.71 \\
\hline $11 / 19 / 1996$ & 235.59 & 733.78 & 256.33 & 733.79 & 271.52 & 733.70 \\
\hline 03/03/1997 & 235.54 & 733.83 & 256.30 & 733.82 & 271.41 & 733.81 \\
\hline 04/15/1997 & 235.63 & 733.74 & 256.40 & 733.72 & 271.54 & 733.68 \\
\hline 06/18/1997 & 235.61 & 733.76 & 256.40 & 733.72 & 271.52 & 733.70 \\
\hline 07/28/1997 & 235.60 & 733.77 & 256.37 & 733.75 & 271.51 & 733.71 \\
\hline 08/20/1997 & 235.52 & 733.85 & 256.29 & 733.83 & 271.44 & 733.78 \\
\hline 09/25/1997 & 235.59 & 733.78 & 256.35 & 733.77 & 271.49 & 733.73 \\
\hline 10/27/1997 & 235.57 & 733.80 & 256.34 & 733.78 & 271.48 & 733.74 \\
\hline 11/03/1997 & 235.65 & 733.72 & 256.40 & 733.72 & 271.55 & 733.67 \\
\hline $11 / 06 / 1997$ & 235.57 & 733.80 & 256.36 & 733.76 & 271.48 & 733.74 \\
\hline $11 / 12 / 1997$ & 235.66 & 733.71 & 256.45 & 733.67 & 271.54 & 733.68 \\
\hline $11 / 13 / 1997$ & 235.60 & 733.77 & 256.29 & 733.83 & 271.49 & 733.73 \\
\hline $11 / 19 / 1997$ & 235.63 & 733.74 & 256.42 & 733.70 & 271.55 & 733.67 \\
\hline $11 / 20 / 1997$ & 235.65 & 733.72 & 256.43 & 733.69 & 271.57 & 733.65 \\
\hline $11 / 25 / 1997$ & 235.64 & 733.73 & 256.39 & 733.73 & 271.54 & 733.68 \\
\hline $11 / 26 / 1997$ & 235.50 & 733.87 & 256.27 & 733.85 & 271.45 & 733.77 \\
\hline $12 / 03 / 1997$ & 235.71 & 733.66 & 256.43 & 733.69 & 271.60 & 733.62 \\
\hline 01/26/1998 & 235.72 & 733.65 & 256.47 & 733.65 & 271.60 & 733.62 \\
\hline 05/12/1998 & 235.60 & 733.77 & 256.32 & 733.80 & 271.52 & 733.70 \\
\hline 10/27/1998 & 235.52 & 733.85 & 256.21 & 733.91 & 271.36 & 733.86 \\
\hline $12 / 22 / 1998$ & 235.54 & 733.83 & 256.20 & 733.92 & 271.35 & 733.87 \\
\hline 02/02/1999 & 235.61 & 733.76 & 256.34 & 733.78 & 271.42 & 733.80 \\
\hline 05/18/1999 & 235.56 & 733.81 & 256.26 & 733.86 & 271.35 & 733.87 \\
\hline 08/25/1999 & 235.56 & 733.81 & 256.26 & 733.86 & 271.38 & 733.84 \\
\hline 10/26/1999 & 235.57 & 733.80 & 256.26 & 733.86 & 271.34 & 733.88 \\
\hline $04 / 24 / 2000$ & 235.64 & 733.73 & 256.34 & 733.78 & 271.52 & 733.70 \\
\hline 08/07/2000 & 235.59 & 733.78 & 256.30 & 733.82 & 271.47 & 733.75 \\
\hline $11 / 13 / 2000$ & 235.66 & 733.71 & 256.34 & 733.78 & 271.45 & 733.77 \\
\hline $02 / 22 / 2001$ & 235.57 & 733.80 & 256.26 & 733.86 & 271.38 & 733.84 \\
\hline 05/21/2001 & 235.67 & 733.70 & 256.35 & 733.77 & 271.49 & 733.73 \\
\hline 08/01/2001 & 235.66 & 733.71 & 256.36 & 733.76 & 271.48 & 733.74 \\
\hline $10 / 01 / 2001$ & 235.66 & 733.71 & 256.35 & 733.77 & 271.45 & 733.77 \\
\hline 02/26/2002 & 235.76 & 733.61 & 256.43 & 733.69 & 271.52 & 733.70 \\
\hline 05/13/2002 & 235.65 & 733.72 & 256.39 & 733.73 & 271.44 & 733.78 \\
\hline 08/19/2002 & 235.61 & 733.76 & 256.28 & 733.84 & 271.42 & 733.80 \\
\hline $10 / 21 / 2002$ & 235.61 & 733.76 & 256.31 & 733.81 & 271.44 & 733.78 \\
\hline $02 / 26 / 2003$ & 235.65 & 733.72 & 256.28 & 733.84 & 271.43 & 733.79 \\
\hline 04/10/2003 & 235.61 & 733.76 & 256.30 & 733.82 & 271.41 & 733.81 \\
\hline 09/10/2003 & 235.74 & 733.63 & 256.35 & 733.77 & 271.50 & 733.72 \\
\hline $10 / 20 / 2003$ & 235.73 & 733.64 & 256.42 & 733.70 & 271.53 & 733.69 \\
\hline $02 / 25 / 2004$ & 235.78 & 733.59 & 256.36 & 733.76 & 271.52 & 733.70 \\
\hline
\end{tabular}




\begin{tabular}{|l|l|l|l|l|l|l|}
$04 / 27 / 2004$ & 235.72 & 733.65 & 256.43 & 733.69 & 271.52 & 733.70 \\
$08 / 18 / 2004$ & 235.72 & 733.65 & 256.38 & 733.74 & 271.48 & 733.74 \\
$10 / 18 / 2004$ & 235.71 & 733.66 & 256.29 & 733.83 & 271.47 & 733.75 \\
$01 / 26 / 2005$ & 235.67 & 733.70 & 256.45 & 733.67 & 271.46 & 733.76 \\
$04 / 18 / 2005$ & 235.66 & 733.71 & 256.33 & 733.79 & 271.44 & 733.78 \\
$07 / 27 / 2005$ & 235.75 & 733.62 & 256.42 & 733.70 & 271.51 & 733.71 \\
$10 / 10 / 2005$ & 235.77 & 733.60 & 256.44 & 733.68 & 271.54 & 733.68 \\
$03 / 08 / 2006$ & 235.74 & 733.63 & 256.39 & 733.73 & 271.50 & 733.72 \\
$05 / 03 / 2006$ & 235.69 & 733.68 & 256.41 & 733.71 & 271.62 & 733.60 \\
$08 / 23 / 2006$ & 235.76 & 733.61 & 256.43 & 733.69 & 271.50 & 733.72 \\
$10 / 09 / 2006$ & 235.69 & 733.68 & 256.38 & 733.74 & 271.44 & 733.78 \\
$02 / 28 / 2007$ & 235.74 & 733.63 & 256.29 & 733.83 & 271.49 & 733.73 \\
$07 / 11 / 2007$ & 235.77 & 733.60 & 256.41 & 733.71 & 271.50 & 733.72 \\
$08 / 28 / 2007$ & 235.78 & 733.59 & 256.42 & 733.70 & 271.47 & 733.75 \\
$10 / 15 / 2007$ & 235.76 & 733.61 & 256.40 & 733.72 & 271.49 & 733.73 \\
$01 / 22 / 2008$ & 235.79 & 733.58 & 256.39 & 733.73 & 271.53 & 733.69 \\
$03 / 03 / 2008$ & 235.80 & 733.57 & 256.38 & 733.74 & 271.53 & 733.69 \\
$06 / 16 / 2008$ & 235.74 & 733.63 & 256.32 & 733.80 & 271.48 & 733.74 \\
$09 / 09 / 2008$ & 235.73 & 733.64 & 256.39 & 733.73 & 271.47 & 733.75 \\
$02 / 17 / 2009$ & 235.78 & 733.59 & 256.40 & 733.72 & 271.52 & 733.70 \\
$05 / 06 / 2009$ & 235.80 & 733.57 & 256.41 & 733.71 & 271.52 & 733.70 \\
$08 / 17 / 2009$ & 235.76 & 733.61 & 256.39 & 733.73 & 271.51 & 733.71 \\
$11 / 10 / 2009$ & 235.81 & 733.56 & 256.46 & 733.66 & 271.55 & 733.67 \\
\hline
\end{tabular}

a Source for northings, eastings, well casing elevations, and deviation corrections: REECO, 1994

${ }^{\mathrm{b}}$ Coordinate System: Nevada (Central) State Plane NAD27

${ }^{\mathrm{c}}$ Measured from top of well casing

${ }^{\mathrm{d}}$ Measured from top of well casing to land surface

Note: All elevations are $m$ above mean sea level

Table 2-11 2009 Area 5 RWMS Groundwater Flow Calculations

\begin{tabular}{|c|c|c|c|}
\hline \multicolumn{4}{|c|}{ Hydraulic Conductivity $=1.12 \mathrm{E}-03 \mathrm{~cm} / \mathrm{s}(3.67 \mathrm{E}-05 \mathrm{ft} / \mathrm{s})^{a}$} \\
\hline \multicolumn{4}{|c|}{ Effective Porosity $=0.38^{\mathrm{a}}$} \\
\hline Date & $\begin{array}{c}\text { Hydraulic } \\
\text { Gradient }(\mathrm{m} / \mathrm{m})\end{array}$ & Velocity (m/yr) & $\begin{array}{c}\text { Flow direction (degrees } \\
\text { East of North) }\end{array}$ \\
\hline 02/17/09 & $9.34 \mathrm{E}-05$ & 0.09 & 194 \\
\hline 05/06/09 & 9.94E-05 & 0.09 & 191 \\
\hline 08/17/09 & 8.64E-05 & 0.08 & 195 \\
\hline $11 / 10 / 09$ & 7.00E-05 & 0.07 & 184 \\
\hline
\end{tabular}

${ }^{a}$ Source: REECO, 1994 


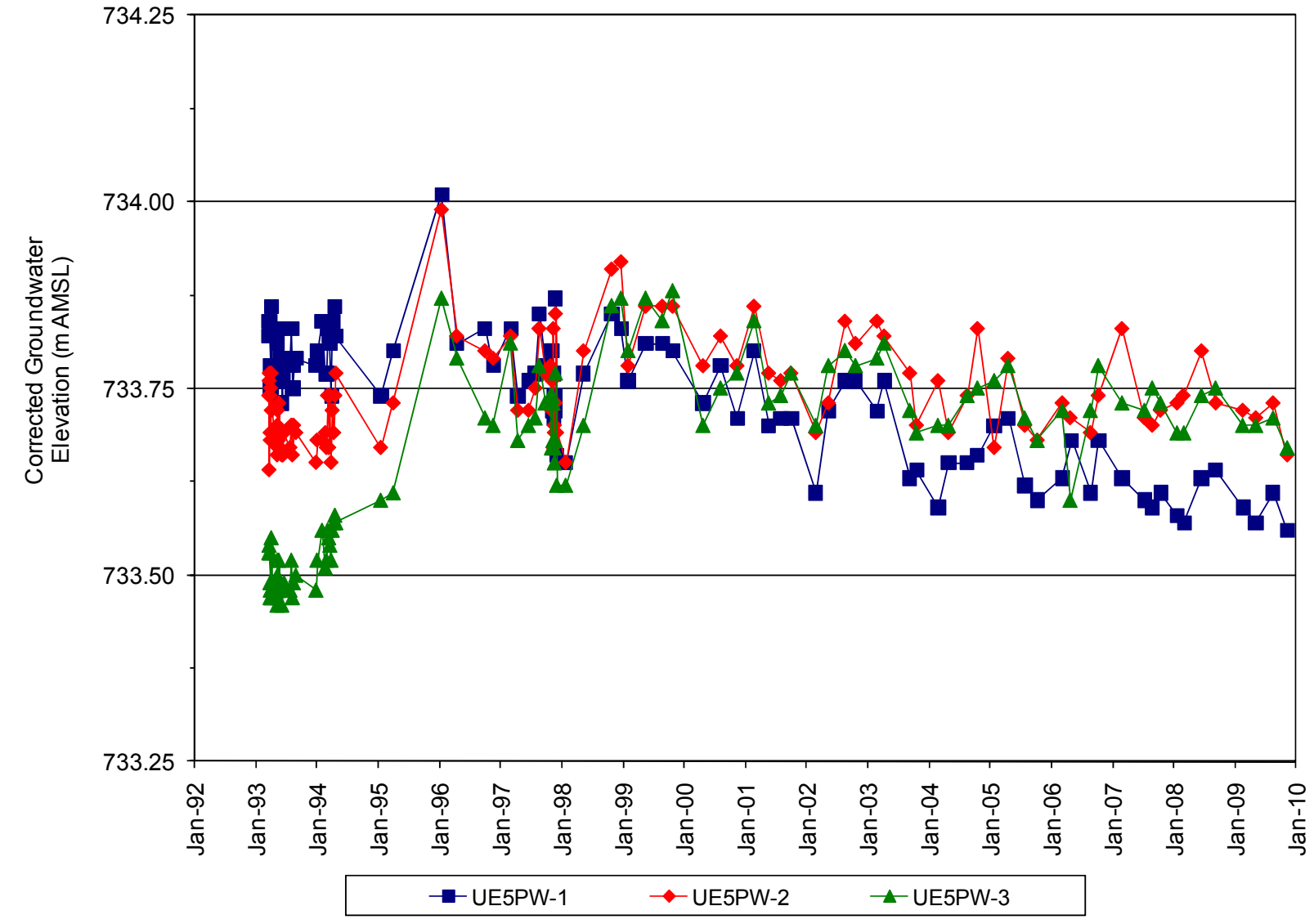

Figure 2-8 Area 5 RWMS Time Series Plot of Groundwater Elevations 
THIS PAGE INTENTIONALLY LEFT BLANK 


\subsection{SUMMARY}

The hydrologic conditions in the uppermost aquifer beneath the Area 5 RWMS remain stable. Groundwater flow in this uppermost aquifer is negligible. No significant changes were detected in the water chemistry, and all indicator parameters remain within the established ILs. 
THIS PAGE INTENTIONALLY LEFT BLANK 


\subsection{CONCLUSION}

There is no measurable impact to the uppermost aquifer from the Area 5 RWMS. 
THIS PAGE INTENTIONALLY LEFT BLANK 


\subsection{REFERENCES}

Bechtel Nevada, 1998. Revised Area 5 Radioactive Waste Management Site, Outline of a Comprehensive Groundwater Monitoring Program. Report to U.S. Department of Energy, Nevada Operations Office. February 1998. Las Vegas, Nevada.

Bechtel Nevada, 2001. Nevada Test Site 2000 Data Report: Groundwater Monitoring Program, Area 5 Radioactive Waste Management Site. Report to U.S. Department of Energy, National Nuclear Security Administration Nevada Site Office, February 2001. DOE/NV/11718--514. Las Vegas, Nevada.

Bechtel Nevada, 2005. A Hydrostratigraphic Framework Model and Alternatives for the Groundwater Flow and Contaminant Transport Model of Corrective Action Unit 98: Frenchman Flat, Clark, Lincoln, and Nye Counties, Nevada. Report to U.S. Department of Energy, National Nuclear Security Administration Nevada Site Office, September 2005. DOE/NV/11718--1064. Las Vegas, Nevada

BN, see Bechtel Nevada.

Bright, D. J., S. A. Watkins, and B. A. Lisle, 2001. Analysis of Water Levels in the Frenchman Flat Area, Nevada Test Site. U.S. Geological Survey Water-Resources Investigations Report 00-4272.

CFR, see Code of Federal Regulations.

Code of Federal Regulations, 1999. Title 40, Part 265, "Interim Status Standards for Owners and Operators of Hazardous Waste Treatment, Storage, and Disposal Facilities."

DOE, see U.S. Department of Energy.

Laczniak, R. J., J. C. Cole, D. A. Sawyer, and D. A. Trudeau, 1996. Summary of the Hydrogeologic Control at the Nevada Test Site, Nye County, Nevada. U.S. Geological Survey Water-Resources Investigations Report 96-4109.

Liebendorfer, P. J., 2000. Nevada Division of Environmental Protection, Carson City, Nevada, Letter to R. C. Wycoff, U.S. Department of Energy, Nevada Operations Office, Las Vegas, Nevada. 1999 Annual Groundwater Monitoring Report, Area 5 Radioactive Waste Management Site (RWMS). April 17, 2000.

National Security Technologies, LLC, 2006. Instructions for Area 5 RWMS Groundwater Well Preparation and Groundwater Sampling. Organization Instruction OI-2154.108. Las Vegas, Nevada.

REECo, see Reynolds Electrical and Engineering Company, Inc.

Reynolds Electrical and Engineering Company, Inc., 1994. Site Characterization and Monitoring Data from Area 5 Pilot Wells, Nevada Test Site, Nye County, Nevada. U.S. Department of Energy Report DOE/NV/11432--74. Las Vegas, Nevada. 
Stoller-Navarro, 2006. Phase II Groundwater Flow Model of Corrective Action Unit 98:

Frenchman Flat, Nevada Test Site, Nye County, Nevada. Report to U.S. Department of Energy, National Nuclear Security Administration Nevada Site Office. May 2006. S-N/99205--074.

U.S. Department of Energy Order 435.1, "Radioactive Waste Management," June 2001.

U.S. Geological Survey, 2009. USGS/US DOE Cooperative Studies in Nevada, Water-Levels Wells Site Map, Nevada Test Site. [Internet] Available at:

http://nevada.usgs.gov/doe nv/ntsmap.htm [Accessed December 16, 2009]. 
Appendix A - Cumulative Chronology for the Area 5 Radioactive Waste Management Site Groundwater Monitoring Program 
THIS PAGE INTENTIONALLY LEFT BLANK 


\begin{tabular}{|c|c|c|c|c|c|}
\hline \multicolumn{6}{|c|}{ Cumulative Chronology for Area 5 RWMS Groundwater (GW) Monitoring Program } \\
\hline Date & UE5PW-1 & Date & UE5PW-2 & Date & UE5PW-3 \\
\hline 03/20/1990 & \multicolumn{5}{|c|}{$\begin{array}{l}\text { U.S. Department of Energy (DOE) letter requesting installation of monitoring wells near the } \\
\text { Area } 5 \text { RWMS. }\end{array}$} \\
\hline 03/13/1992 & \multicolumn{5}{|l|}{ Drilling begins } \\
\hline 06/16/1992 & Drilling ends & 06/18/1992 & Drilling begins & & \\
\hline \multirow[t]{4}{*}{ 09/11/1992 } & Well developed & 09/04/1992 & Drilling ends & \multirow[b]{2}{*}{$09 / 16 / 1992$} & \\
\hline & & & & & Drilling begins \\
\hline & & & & 11/09/1992 & Drilling ends \\
\hline & & 03/24/1993 & GW Sampling & & \\
\hline \multirow[t]{2}{*}{ 03/31/1993 } & GW Sampling & 03/30/1993 & Well developed & 04/04/1993 & Well developed \\
\hline & & & & 04/14/1993 & GW Sampling \\
\hline 06/06/1993 & GW Sampling & 06/22/1993 & GW Sampling & 06/02/1993 & GW Sampling \\
\hline 09/01/1993 & GW Sampling & & & 10/12/1993 & GW Sampling \\
\hline 12/07/1993 & GW Sampling & 11/15/1993 & GW Sampling & $12 / 20 / 1993$ & GW Sampling \\
\hline 12/17/1993 & \multicolumn{5}{|c|}{$\begin{array}{l}\text { DOE letter to Nevada Department of Environmental Protection (NDEP) requesting to } \\
\text { establish pilot wells located near the Area } 5 \text { RWMS as Resource Conservation and } \\
\text { Recovery Act (RCRA) GW monitoring wells. }\end{array}$} \\
\hline $02 / 24 / 1994$ & \multicolumn{5}{|c|}{$\begin{array}{l}\text { NDEP letter stating that the pilot wells appear to meet the applicable design, construction, } \\
\text { and development criteria for RCRA GW monitoring wells. }\end{array}$} \\
\hline 06/15/1994 & GW Sampling & 06/07/1994 & GW Sampling & 05/24/1994 & GW Sampling \\
\hline \multirow[t]{2}{*}{ 08/01/1994 } & GW Sampling & & & 08/08/1994 & GW Sampling \\
\hline & & 11/29/1994 & GW Sampling & & \\
\hline 09/30/1994 & \multicolumn{5}{|c|}{ DOE submits $1993 \mathrm{GW}$ monitoring results from quarterly sampling effort. } \\
\hline 01/18/1995 & \multicolumn{5}{|c|}{ UE5PW-3 GW resampling for 08/01/1994 total organic carbon (TOC) hit. } \\
\hline 02/23/1995 & \multicolumn{5}{|c|}{ DOE transmits to NDEP GW Monitoring Program Outline. } \\
\hline $03 / 01 / 1995$ & \multicolumn{5}{|c|}{1994 GW Monitoring Report submitted to NDEP. } \\
\hline 04/04/1995 & \multicolumn{5}{|l|}{ GW Sampling } \\
\hline $11 / 09 / 1995$ & \multicolumn{5}{|l|}{ GW Sampling } \\
\hline $11 / 09 / 1995$ & \multicolumn{5}{|c|}{ UE5PW-1 pump snagged in hole, resulting in a bent shaft on the reel. } \\
\hline $01 / 18 / 1996$ & GW Sampling & $01 / 25 / 1996$ & GW Sampling & $01 / 18 / 1996$ & GW Sampling \\
\hline $01 / 22 / 1996$ & \multicolumn{5}{|c|}{ Bennett pump seals replaced at all three wells. } \\
\hline
\end{tabular}




\begin{tabular}{|c|c|c|c|c|}
\hline \multicolumn{5}{|c|}{ Cumulative Chronology for Area 5 RWMS Groundwater (GW) Monitoring Program } \\
\hline Date & UE5PW-1 & Date & UE5PW-2 & UE5PW-3 \\
\hline 03/01/1996 & \multicolumn{4}{|c|}{ DOE submits to NDEP the 1995 GW Monitoring Report. } \\
\hline 04/16/1996 & GW Sampling & $04 / 23 / 1996$ & \multicolumn{2}{|l|}{ GW Sampling } \\
\hline & & $04 / 30 / 1996$ & \multicolumn{2}{|l|}{ GW Sampling } \\
\hline 10/02/1996 & \multicolumn{4}{|l|}{ GW Sampling } \\
\hline 10/25/1996 & \multicolumn{4}{|c|}{ NDEP requests clarifications/changes in the GW Monitoring Report. } \\
\hline 11/20/1996 & \multicolumn{4}{|l|}{ GW Sampling } \\
\hline 03/01/1997 & \multicolumn{4}{|c|}{ DOE submits 1996 GW Monitoring Report and revised GW Monitoring Program Outline. } \\
\hline 04/16/1997 & \multicolumn{4}{|c|}{ GW Sampling } \\
\hline 08/12/1997 & \multicolumn{4}{|c|}{ NDEP comments on 1996 GW Monitoring Report/Proposed Outline. } \\
\hline 10/22/1997 & \multicolumn{4}{|c|}{$\begin{array}{l}\text { Pump and water-level meter lodge in UE5PW-1 during simultaneous operation, retrieved } \\
10 / 23 / 1997 .\end{array}$} \\
\hline 10/22/1997 & \multicolumn{4}{|c|}{ Larger diameter air lines installed at all three wells. } \\
\hline $11 / 05 / 1997$ & \multicolumn{4}{|l|}{ GW Sampling } \\
\hline $03 / 01 / 1998$ & \multicolumn{4}{|c|}{ DOE submits to NDEP the 1997 GW Monitoring Report and new outline. } \\
\hline 03/31/1998 & \multicolumn{4}{|c|}{$\begin{array}{l}\text { NDEP letter stating that they concur on the indicator parameters and investigation levels } \\
\text { submitted in the GW Monitoring Outline. }\end{array}$} \\
\hline 05/13/1998 & \multicolumn{4}{|l|}{ GW Sampling } \\
\hline 06/22/1998 & \multicolumn{4}{|c|}{$\begin{array}{l}\text { Total organic halides (TOX) detected in the } 05 / 13 / 1998 \text { samples and blanks from all three } \\
\text { wells. }\end{array}$} \\
\hline 07/10/1998 & \multicolumn{4}{|c|}{ DOE and NDEP agree to resample UE5PW-1 to confirm no TOX. } \\
\hline 07/29/1998 & \multicolumn{4}{|c|}{ GW resampling at UE5PW-1 for 05/13/1998 TOX hits. } \\
\hline 09/10/1998 & \multicolumn{4}{|c|}{$\begin{array}{l}\text { Results from 07/29/1998 resampling are non-detect for TOX. TOX results from the } \\
05 / 13 / 1998 \text { sampling event are determined to be false positives. }\end{array}$} \\
\hline 09/10/1998 & \multicolumn{4}{|c|}{ Bennett pumps from three wells and spare pumps are sent to manufacturer for refurbishing. } \\
\hline 09/12/1998 & \multicolumn{4}{|c|}{ Reels from three wells are returned to manufacturer for new tubing bundles. } \\
\hline 10/28/1998 & \multicolumn{4}{|l|}{ GW Sampling } \\
\hline 09/12/1998 & \multicolumn{4}{|c|}{$\begin{array}{l}\text { UE5PW-1 reel returned to manufacturer for repair of exhaust tube. Spare pump returned to } \\
\text { manufacturer for the repair of a leaky seal. }\end{array}$} \\
\hline 03/01/1999 & \multicolumn{4}{|c|}{ DOE submits to NDEP 1998 GW Monitoring Report. } \\
\hline 03/31/1999 & \multicolumn{4}{|c|}{$\begin{array}{l}\text { NDEP requests statistical analysis of data and states that values determined to be false } \\
\text { positives through resampling do not need to be presented graphically. }\end{array}$} \\
\hline 05/19/1999 & \multicolumn{4}{|l|}{ GW Sampling } \\
\hline 10/27/1999 & \multicolumn{4}{|l|}{ GW Sampling } \\
\hline 12/13/1999 & \multicolumn{4}{|c|}{ Resample UE5PW-2 after TOC hit from 10/27/1999. } \\
\hline
\end{tabular}




\begin{tabular}{|c|c|c|c|c|c|}
\hline \multicolumn{6}{|c|}{ Cumulative Chronology for Area 5 RWMS Groundwater (GW) Monitoring Program } \\
\hline Date & UE5PW-1 & Date & UE5PW-2 & Date & UE5PW-3 \\
\hline $12 / 27 / 1999$ & \multicolumn{5}{|c|}{$\begin{array}{l}\text { Results from the resampling of UE5PW-2 are non-detect for TOC. TOC result from } \\
10 / 27 / 1999 \text { is determined to be a false positive. }\end{array}$} \\
\hline $02 / 25 / 2000$ & \multicolumn{5}{|c|}{ DOE submits to NDEP 1999 GW Monitoring Report. } \\
\hline $04 / 17 / 2000$ & \multicolumn{5}{|c|}{ NDEP states that future reports do not need to include statistical analyses. } \\
\hline $04 / 26 / 2000$ & GW Sampling & & & & \\
\hline $06 / 28 / 2000$ & \multicolumn{5}{|c|}{$\begin{array}{l}\text { DOE contacts State to report TOX/TOC hits from 04/26/2000. DOE and NDEP agree that } \\
\text { the wells will be resampled in August, which would also constitute the Fall sampling event. }\end{array}$} \\
\hline $08 / 09 / 2000$ & GW Sampling & & & & \\
\hline $09 / 20 / 2000$ & \multicolumn{5}{|c|}{ DOE contacts NDEP to report TOX hits from 08/09/2000 sampling. } \\
\hline $11 / 07 / 2000$ & \multicolumn{5}{|c|}{$\begin{array}{l}\text { Letter from NDEP stating that DOE does not have a valid data set for TOX and possibly } \\
\text { TOC and requests a plan to address contamination concerns prior to next sampling event. }\end{array}$} \\
\hline $11 / 20 / 2000$ & Video log well & & & $11 / 27 / 2000$ & Video log well \\
\hline $12 / 20 / 2000$ & \multicolumn{5}{|c|}{ DOE transmits to NDEP a proposed plan to address contamination issues. } \\
\hline $01 / 31 / 2001$ & \multicolumn{5}{|c|}{$\begin{array}{l}\text { Letter from NDEP generally concurring that the plan submitted to determine the cause of } \\
\text { TOX and TOC hits is sound. }\end{array}$} \\
\hline $02 / 21 / 2001$ & \multicolumn{5}{|c|}{ DOE submits to NDEP 2000 GW Monitoring Report } \\
\hline $03 / 14 / 2001$ & \multicolumn{5}{|c|}{$\begin{array}{l}\text { Letter from NDEP stating that the } 2000 \mathrm{GW} \text { Monitoring Report was received in a timely } \\
\text { manner and contains all the data required by Title } 40 \text { Code of Federal Regulations } \\
\text { Part 265.94. Letter also requests information regarding data in Appendix A of the } 2000 \mathrm{GW} \\
\text { Monitoring Report (Bechtel Nevada, 2001). }\end{array}$} \\
\hline $04 / 19 / 2001$ & \multicolumn{5}{|c|}{$\begin{array}{l}\text { Letter from DOE responding to NDEP's } 3 / 14 / 2001 \text { request for information regarding } \\
\text { presentation of TOX/TOC data in the } 2000 \text { report. }\end{array}$} \\
\hline $04 / 30 / 2001$ & \multicolumn{5}{|c|}{$\begin{array}{l}\text { Letter from NDEP concurring with the approach to data presentation as outlined by DOE in } \\
\text { the } 4 / 19 / 2001 \text { correspondence. }\end{array}$} \\
\hline $05 / 29 / 2001$ & \multicolumn{5}{|l|}{ GW Sampling } \\
\hline $10 / 03 / 2001$ & \multicolumn{5}{|l|}{ GW Sampling } \\
\hline $03 / 01 / 2002$ & \multicolumn{5}{|c|}{ DOE submits to NDEP 2001 GW Monitoring Report. } \\
\hline $05 / 15 / 2002$ & \multicolumn{5}{|l|}{ GW Sampling } \\
\hline $10 / 22 / 2002$ & \multicolumn{5}{|l|}{ GW Sampling } \\
\hline $03 / 01 / 2003$ & \multicolumn{5}{|c|}{ DOE submits to NDEP 2002 GW Monitoring Report. } \\
\hline $04 / 15 / 2003$ & \multicolumn{5}{|l|}{ GW Sampling } \\
\hline $10 / 22 / 2003$ & \multicolumn{3}{|l|}{ GW Sampling } & $10 / 21 / 2003$ & GW Sampling \\
\hline
\end{tabular}




\begin{tabular}{|c|c|c|c|c|c|}
\hline \multicolumn{6}{|c|}{ Cumulative Chronology for Area 5 RWMS Groundwater (GW) Monitoring Program } \\
\hline Date & UE5PW-1 & Date & UE5PW-2 & Date & UE5PW-3 \\
\hline 02/27/2004 & \multicolumn{5}{|c|}{ DOE submits to NDEP 2003 GW Monitoring Report. } \\
\hline 05/04/2004 & \multicolumn{5}{|l|}{ GW Sampling } \\
\hline 10/19/2004 & \multicolumn{3}{|l|}{ GW Sampling } & $10 / 20 / 2003$ & GW Sampling \\
\hline $02 / 25 / 2005$ & \multicolumn{5}{|c|}{ DOE submits to NDEP 2004 GW Monitoring Report. } \\
\hline 04/19/2005 & \multicolumn{5}{|l|}{ GW Sampling } \\
\hline $10 / 11 / 2005$ & \multicolumn{5}{|l|}{ GW Sampling } \\
\hline 02/28/2006 & \multicolumn{5}{|c|}{ DOE submits to NDEP 2005 GW Monitoring Report. } \\
\hline 04/26/2006 & \multicolumn{5}{|l|}{ GW Sampling } \\
\hline 10/10/2006 & \multicolumn{5}{|l|}{ GW Sampling } \\
\hline 03/01/2007 & \multicolumn{5}{|c|}{ DOE submits to NDEP 2006 GW Monitoring Report. } \\
\hline 03/19/2007 & \multicolumn{5}{|l|}{ GW Sampling } \\
\hline 08/29/2007 & \multicolumn{3}{|l|}{ GW Sampling } & $09 / 05 / 2007$ & GW Sampling \\
\hline 03/01/2008 & \multicolumn{5}{|c|}{ DOE submits to NDEP 2007 GW Monitoring Report } \\
\hline $03 / 11 / 2008$ & \multicolumn{5}{|l|}{ GW Sampling } \\
\hline 09/10/2008 & \multicolumn{5}{|l|}{ GW Sampling } \\
\hline 04/22/2009 & \multicolumn{5}{|c|}{ DOE submits to NDEP 2008 GW Monitoring Report } \\
\hline $03 / 10 / 2009$ & \multicolumn{5}{|l|}{ GW Sampling } \\
\hline 08/18/2009 & \multicolumn{5}{|l|}{ GW Sampling } \\
\hline
\end{tabular}




\section{Appendix B - Gradient/Velocity Calculations}


THIS PAGE INTENTIONALLY LEFT BLANK 


\section{Calculation of Magnitude and Direction of Area 5 Alluvial Aquifer Gradient}

Water level elevations measured at three wells near the Area 5 Radioactive Waste Management Site (UE5PW-1, UE5PW-2, and UE5PW-3) are used to calculate the magnitude and direction of the aquifer hydraulic gradient.

The locations of the three wells are given in Nevada State Central Zone coordinates in meters as northing $(N)$ and easting $(E)$ values. The coordinates of each of the three water elevation points define a plane that contains the water level points. The coordinates of the water elevation points are $\left(\mathrm{E}_{\mathrm{i}}, \mathrm{N}_{\mathrm{i}}, \mathrm{e}_{\mathrm{i}}\right)$ where:

$E_{i}$ is the East Coordinate of the $i^{\text {th }}$ well $(m)$

$\mathrm{N}_{\mathrm{i}}$ is the North Coordinate of the $\mathrm{i}^{\text {th }}$ well $(\mathrm{m})$

$\mathrm{e}_{i}$ is the water level elevation of the $\mathrm{i}^{\mathrm{th}}$ well $(\mathrm{m})$

Assuming $\mathrm{i}=1$ for UE5PW-1, $\mathrm{i}=2$ for UE5PW-2, and $\mathrm{i}=3$ for UE5PW-3, the vector a connecting the water level at UE5PW-1 to the water level at UE5PW-2 and the vector $\mathbf{b}$ connecting the water level at UE5PW-1 to the water level at UE5PW-3 are defined by:

$$
\begin{aligned}
& \mathbf{a}=\left(E_{2}-E_{1}\right) \mathbf{i}+\left(N_{2}-N_{1}\right) \mathbf{j}+\left(e_{2}-e_{1}\right) \mathbf{k} \\
& \mathbf{b}=\left(E_{3}-E_{1}\right) \mathbf{i}+\left(N_{3}-N_{1}\right) \mathbf{j}+\left(e_{3}-e_{1}\right) \mathbf{k}
\end{aligned}
$$

The aquifer hydraulic gradient is the cross product $\mathbf{a} \times \mathbf{b}$.

$$
\begin{array}{cccc}
\boldsymbol{a} \boldsymbol{x} \boldsymbol{b}=\boldsymbol{D E T}\left[\begin{array}{ccc}
\boldsymbol{i} & \boldsymbol{j} & \boldsymbol{k} \\
\boldsymbol{E}_{2}-E_{1} & N_{2}-N_{1} & e_{2}-e_{1} \\
E_{3}-E_{1} & N_{3}-N_{1} & e_{3}-e_{1}
\end{array}\right] \\
= & {\left[\left(\mathrm{N}_{2}-\mathrm{N}_{1}\right)\left(\mathrm{e}_{3}-\mathrm{e}_{1}\right)-\left(\mathrm{e}_{2}-\mathrm{e}_{1}\right)\left(\mathrm{N}_{3}-\mathrm{N}_{1}\right)\right] \mathbf{i}+} \\
& {\left[\left(\mathrm{e}_{2}-\mathrm{e}_{1}\right)\left(\mathrm{E}_{3}-\mathrm{E}_{1}\right)-\left(\mathrm{E}_{2}-\mathrm{E}_{1}\right)\left(\mathrm{e}_{3}-\mathrm{e}_{1}\right)\right] \mathbf{j}+} \\
& {\left[\left(\mathrm{E}_{2}-\mathrm{E}_{1}\right)\left(\mathrm{N}_{3}-\mathrm{N}_{1}\right)-\left(\mathrm{N}_{2}-\mathrm{N}_{1}\right)\left(\mathrm{E}_{3}-\mathrm{E}_{1}\right)\right] \mathbf{k}} \\
= & \mathrm{Ai}+\mathrm{Bj}+\mathrm{Ck}
\end{array}
$$

Where: $A=\left(N_{2}-N_{1}\right)\left(e_{3}-e_{1}\right)-\left(e_{2}-e_{1}\right)\left(N_{3}-N_{1}\right)$

$B=\left(e_{2}-e_{1}\right)\left(E_{3}-E_{1}\right)-\left(E_{2}-E_{1}\right)\left(e_{3}-e_{1}\right)$

$C=\left(E_{2}-E_{1}\right)\left(N_{3}-N_{1}\right)-\left(N_{2}-N_{1}\right)\left(E_{3}-E_{1}\right)$

Dividing hydraulic gradient by $\mathrm{C}$ gives the magnitude of the gradient in Easting (i) and Northing (j) for a unit change in elevation (k)

$$
(\boldsymbol{a} \times \boldsymbol{b}) / C=A / C \boldsymbol{i}+B / C \boldsymbol{j}+\boldsymbol{k}
$$


The magnitude of the gradient is:

$$
\sqrt{A / C^{2}+B / C^{2}}
$$

The direction of the gradient from north $(\theta)$ is calculated using the magnitudes of easting $(E)$ and northing $(\mathrm{N})$.

If $B>0$, then $\theta=\arctan (a / b)$

If $B<0$, then $\theta=180^{\circ}+\arctan (a / b)$

If $B=0$ and $A>0$, then $\theta=90^{\circ}$

If $B=0$ and $A<0$, then $\theta=270^{\circ}$

If $B=0$ and $A=0$, then the flow is straight down.

\section{Calculation of Mean Groundwater Velocity}

Groundwater flux is from calculated from Darcy's Law:

Where: $\quad J$ is groundwater flux $(\mathrm{m} / \mathrm{s})$

$$
J=-K\left(\frac{\Delta e}{C}\right)
$$

$\mathrm{K}$ is saturated hydraulic conductivity $(\mathrm{m} / \mathrm{s})$

$\frac{\Delta e}{C}$ is the hydraulic gradient $(\mathrm{m} / \mathrm{m})$

The mean groundwater velocity is calculated from the flux:

$$
\boldsymbol{V}=\boldsymbol{J} / \varnothing
$$

Where: $\quad V$ is mean groundwater velocity $(\mathrm{m} / \mathrm{s})$

$J$ is the groundwater flux $(\mathrm{m} / \mathrm{s})$

$\Phi$ is porosity $\left(\mathrm{m}^{3} / \mathrm{m}^{3}\right)$ 


\section{DISTRIBUTION LIST}

\section{U. S. Department of Energy, National Nuclear Security Administration Nevada Site Office}

Jhon T. Carilli

4

LLW Federal Sub-Project Director, Waste Management Project

U.S. Department of Energy

National Nuclear Security Administration

Nevada Site Office

P.O. Box $98518 \mathrm{M} / \mathrm{S} 505$

Las Vegas, NV 89193-8518

E. Frank Di Sanza

1

Federal Project Director, Waste Management Project

U.S. Department of Energy

National Nuclear Security Administration

Nevada Site Office

P.O. Box $98518 \mathrm{M} / \mathrm{S} 505$

Las Vegas, NV 89193-8518

Kenneth M. Small

1

RCRA Program Manager, Waste Management Project

U.S. Department of Energy

National Nuclear Security Administration

Nevada Site Office

P.O. Box 98518 M/S 505

Las Vegas, NV 89193-8518

U.S. Department of Energy

2 (CD)

National Nuclear Security Administration

Nevada Site Office

Public Reading Facility

P.O. Box 98521, M/S 400

Las Vegas, NV 89193-8521

U.S. Department of Energy

National Nuclear Security Administration

$1(C D)$

Nevada Site Office

Technical Library

P.O. Box 98518, M/S 505

Las Vegas, NV 89193-8518

U.S Department of Energy

Office of Scientific and Technical Information

1 digital file

P.O. Box 62

Oak Ridge, TN 37831-0062 
National Security Technologies, LLC

Environmental Management Library

National Security Technologies, LLC

P.O. Box 98518, M/S NLV094

Las Vegas, NV 89193-8521

David B. Hudson

National Security Technologies, LLC

P.O. Box 98521, M/S NLV083

Las Vegas, NV 89193-8521

Theodore J. Redding

National Security Technologies, LLC

P.O. Box 98521, M/S NTS273

Las Vegas, NV 89193-8521

Gregory J. Shott

National Security Technologies, LLC

P.O. Box 98521, M/S NLV083

Las Vegas, NV 89193-8521

Vefa Yucel

National Security Technologies, LLC

P.O. Box 98521, M/S NLV083

Las Vegas, NV 89193-8521 